\title{
Galactic dynamos with captured magnetic flux and an accretion flow
}

\author{
D. Moss ${ }^{1}$ and A. Shukurov ${ }^{2}$ \\ 1 Department of Mathematics, University of Manchester, Manchester M13 9PL, UK \\ 2 Department of Mathematics, University of Newcastle, Newcastle upon Tyne NE1 7RU, UK
}

Received 15 December 2000 / Accepted 2 April 2001

\begin{abstract}
We examine the behaviour of an axisymmetric galactic dynamo model with a radial accretion flow in the disc. We also introduce a vertical magnetic flux through the galactic midplane, to simulate the presence of a large scale magnetic field trapped by the galaxy when forming. The trapped vertical flux is conserved and advected towards the disc centre by the radial flow. We confirm that accretion flows of magnitude several $\mathrm{km} \mathrm{s}^{-1}$ through a significant part of the galactic disc can markedly inhibit dynamo action. Moreover, advection of the vertical flux in general results in mixed parity galactic fields. However, the effect is nonlinear and non-additive - global magnetic field energies are usually significantly smaller that the sum of purely dynamo generated and purely advected field energies. For large inflow speeds, a form of "semi-dynamo" action may occur. We apply our results to the accumulation and redistribution, by a radial inflow, of a vertical magnetic flux captured by the Galactic disc. Taking representative values, it appears difficult to obtain mean vertical fields near the centre of the Milky Way that are much in excess of $10 \mu \mathrm{G}$, largely because the galactic dynamo and turbulent magnetic diffusion modify the external magnetic field before it can reach the disc centre.
\end{abstract}

Key words. magnetic fields - MHD - ISM: magnetic fields - galaxies: ISM - galaxies: magnetic fields - galaxies: spiral

\section{Introduction}

Dynamo systems only rarely occur isolated magnetically from their environment. To assume such isolation may be a plausible assumption for, e.g., stellar dynamos, but the situation is not so clearcut for spiral galaxies. Nevertheless, it is generally supposed that the dynamo acts independently of any trapped vertical flux, and the interaction of a dynamo system with its magnetic environment has rarely been addressed. However, the effects of external magnetic fields have been invoked to explain the origins of various features of astrophysical discs, such as vertical dust filaments in spiral galaxies (Sofue 1987), strong vertical magnetic fields in the centre of the Milky Way (Sofue \& Fujimoto 1987; Chandran et al. 2000) and, in a different astrophysical (but not conceptual) context, the launching and collimation of outflows in accretion discs around young stars and compact objects (Blandford \& Payne 1982; Mestel 1999). It is widely accepted, however, that both accretion discs and the discs of spiral galaxies are sites of dynamo action (Parker 1979; Zeldovich et al. 1983; Ruzmaikin et al. 1988; Beck et al. 1996; Kulsrud 1999; Mestel 1999). In this paper we discuss, in a galactic

Send offprint requests to: D. Moss, e-mail: moss@ma.man.ac.uk context, the nonlinear interaction between a disc dynamo and a trapped vertical flux redistributed by the radial inflow of matter.

The most important aspect of the problem in the galactic context is the origin of the vertical magnetic field in the Galactic centre where it can be as strong as $1 \mathrm{mG}$ within the inner $200 \mathrm{pc}$ radius, implying vertical magnetic flux of about $40 \mathrm{G} \mathrm{pc}^{2}$ if the field is unidirectional and pervasive (Morris \& Serabyn 1996). The field geometry is apparently dipole-like, in contrast to the quadrupole symmetry dominant near the Sun. With a gas density of $10-100 \mathrm{~cm}^{-3}$ between molecular clouds and the turbulent velocities of $20-30 \mathrm{~km} \mathrm{~s}^{-1}$ (e.g., Morris \& Serabyn 1996), the field is 100-10 times above the equipartition level with the turbulent kinetic energy. These features suggest that the vertical magnetic field in the Galactic centre might arise from an accumulation of an external magnetic flux advected from large radii over the Galactic lifetime (Sofue \& Fujimoto 1987; Chandran et al. 2000). However, the Galactic disc is magnetically active, being a site of dynamo action, and so it is not clear whether or not any vertical magnetic field can be dragged through it. Our results suggest that it is rather implausible that the vertical magnetic flux at the 
Galactic centre results from the redistribution of magnetic field trapped at an early stage of Galactic evolution.

\section{Formulation of the problem}

The interaction of a nonlinear dynamo system with an external magnetic field can be non-trivial. Drobyshevski (1977) proposed the idea of the "semi-dynamo", a system which amplifies magnetic field at the expense of kinetic energy like a self-excited dynamo, but in which the field would decay without the presence of an external magnetic field. Convective dynamos in the presence of an imposed magnetic field have been studied by Sarson et al. (1997) and Sarson et al. (1999) in application to magnetic fields of Io and Ganymede, Jovian satellites orbiting within the giant planet's magnetosphere. These authors report on magnetic states with self-maintained magnetic field which, however, revert to a state of nonmagnetic convection if the external field is withdrawn or weakened. In this model, a uniform magnetic field parallel to the rotation axis is included as a source term in the induction equation, although the results remain the same when the external magnetic field is introduced through boundary conditions (G. R. Sarson, private communication). There is a plausible connection between the behaviour of these systems and that of Drobyshevski's semi-dynamos.

There have been a number of studies of stellar dynamos with a fixed magnetic field as boundary condition at the bottom of the convection zone, simulating the presence of an interior field frozen in to the radiative core (e.g. Pudovkin \& Benevolenskaya 1984; Boyer \& Levy 1984; Moss 1996). The effect here was less fundamental, being to change properties such as parity, rather than cause semidynamo action.

A two-dimensional mean-field dynamo with external magnetic field has been considered by Reyes-Ruiz \& Stepinski (1997) who, however, neglected any radial velocity and obtained a solution of the nonlinear ( $\alpha$-quenched) dynamo equations with external magnetic field by merely adding a constant vertical field to the solution of the standard dynamo equations. The physical importance of their results for realistic degrees of nonlinearity is therefore questionable.

The inward advection (dragging) of an external magnetic field by accretion flow in a Keplerian disc has been considered by Lubow et al. (1994) (numerical solutions have been presented by Reyes-Ruiz \& Stepinski 1996). These authors disregarded any dynamo action and considered only the balance between advection of a purely poloidal magnetic field and radial diffusion. Their results show that efficient advection of an external magnetic field into a viscous disc is only possible if the magnetic diffusivity is much smaller than the kinetic. Otherwise inward advection is balanced by radial magnetic diffusion and the steady-state magnetic field in the disc can only be weak.

There are several possible types of external magnetic field for galaxies and accretion discs. This can be a field of cosmological origin. Current models of magnetic field generation in the early Universe predict that the strength of a primeval field at the galactic scales can only be negligible (e.g. Beck et al. 1996), so this option has lost its importance. For galaxies belonging to a galaxy cluster (but not the Milky Way), the intracluster magnetic field (Kronberg 1994) can be a significant source of external magnetic flux. The strength of this field is about $1 \mu \mathrm{G}$, but only a fraction of it may have the appropriate poloidal structure in the galactic frame. Away from rich galaxy clusters, the observational upper limit on the cosmological magnetic field $\left(<3 \times 10^{-12} \mathrm{G}\right.$, Sciama 1994; Beck et al. 1996) implies an upper limit of $3 \times 10^{-10} \mathrm{G}$ for a quasi-uniform magnetic field captured by and compressed in a collapsing protogalaxy (for radial compression by a factor 10). For accretion discs in an active galactic nucleus or in a stellar system, the interstellar magnetic field of the parent galaxy ( $\simeq 1 \mu \mathrm{G}$ for the total field of which a fraction would be poloidal field as seen by the disc) can serve as an external field. Stronger external magnetic fields originating in the central star can occur in accretion discs of binary stellar systems (e.g. Bardou \& Heyvaerts 1996).

Although the relative orientation of the disc plane and the external magnetic field can be arbitrary, we neglect any magnetic field component parallel to the disc plane and allow only for a component parallel to the rotation axis. Any horizontal component will be twisted by differential rotation until the separation of magnetic line segments of alternating sign becomes small enough for the field to be destroyed by turbulent magnetic diffusion and/or reconnection (e.g. Sects. 3.8 and 3.9 of Moffatt 1978). The only way to avoid or postpone the expulsion is by reducing the magnetic diffusion as, e.g., in Howard \& Kulsrud (1997) and Chandran et al. (2000), who only consider the effects of a relatively weak ambipolar diffusivity. Observations directly indicate the presence of intense turbulent motions in the interstellar gas of spiral galaxies (Minter \& Spangler 1996). Even though interstellar magnetic fields are dynamically important, there are no apparent indications of any modification of the turbulence that could result in any significant suppression of turbulent diffusion. Furthermore, Howard \& Kulsrud (1997) neglect magnetic buoyancy, which can be efficient in removing horizontal magnetic field from the disc on a time scale of order the Alfvén crossing time over the disc scale height. Magnetic buoyancy possibly presents insurmountable difficulties for a horizontal primordial magnetic field, whereas galactic dynamo action may even be enhanced by buoyancy (Parker 1992; Moss et al. 1999); however we neglect the effects of magnetic buoyancy here so as to keep the model as simple as possible.

Thus, any horizontal external magnetic field will be unimportant. However, the behaviour of the vertical magnetic field $B_{z}$ is different. For a mean velocity field (including rotation) that is independent of the vertical coordinate $z$, a uniform $B_{z}$ is not distorted by the flow and is not subject to vertical diffusion (since $\partial^{2} B_{z} / \partial z^{2} \equiv 0$ ). Therefore, such a field can be dragged into the disc by a radial flow. The dragging is especially efficient if the disc 
is surrounded by vacuum (where magnetic diffusivity is effectively infinite). The pile-up of the vertical magnetic field will be opposed and balanced by the radial magnetic diffusion. The resulting steady state was discussed by Lubow et al. (1994). A $z$-dependent rotation would add an axisymmetric azimuthal magnetic field produced from $B_{z}$ by the vertical shear. In addition, an azimuthal field can be produced by differential rotation from a radial field generated by a vertical gradient in the inflow velocity.

This picture is very much enriched by any dynamo action in the disc. The dynamo generates its own magnetic field that can interact with the intruding vertical flux either constructively or destructively. The basic dynamo mode in a galactic disc (where the $\alpha$-effect is positive in the northern hemisphere) is of quadrupolar parity (see, e.g., Parker 1979; Zeldovich et al. 1983; Ruzmaikin et al. 1988), opposite to the parity of the (quasi-)uniform vertical magnetic field. Therefore, the two fields can only interact via nonlinear effects. The situation can be different in accretion discs where magneto-rotational instability can produce a negative $\alpha$-effect resulting in a predominantly dipolar dynamo field (Brandenburg et al. 1995). The synergy of the vertical flux and the dynamo may result in physically nontrivial effects such as the semidynamo of Drobyshevski. Some of the behaviours observed in our models for large inflow speed can be considered as a nonlinear analogue of semi-dynamo action wherein a subcritical dynamo action modifies non-trivially an invading external magnetic field.

Radial flows can affect the disc dynamo in two obvious ways, both suppressive. As discussed by Moss et al. (2000), the radial advection hinders dynamo action, basically because the advection distorts the field from the eigenfunction. This effect can be important when the radial velocity $u_{\mathrm{r}} \gtrsim 2 \eta / h \simeq 1 \mathrm{~km} \mathrm{~s}^{-1}$, for parameter values typical of the Solar neighbourhood $(h \simeq 500 \mathrm{pc}$ is the scale height of the ionized layer, $\eta \simeq 10^{26} \mathrm{~cm}^{2} \mathrm{~s}^{-1}$ is the turbulent magnetic diffusivity). The second effect is due to the contribution of an imposed magnetic flux, dragged by the flow, to the nonlinear saturation of the dynamo action, customarily modelled by the $\alpha$-quenching. Now the strength of the $\alpha$-effect, responsible for the dynamo action, is reduced by the magnetic field as $\alpha \propto\left(1+B^{2} / B_{0}^{2}\right)^{-1}$, where $B$ is the strength of the local mean magnetic field, comprising both the dynamo and advected imposed flux. Here $B_{0}$ is a reference field strength at which nonlinear dynamo effects become pronounced. The suppression of the dynamo action by the intruding magnetic field can be expected to be significant if it becomes comparable to $B_{0}$.

All these effects cannot easily be separated, and it is difficult to assess their possible importance without the detailed analysis attempted here. In particular, our results presented below show that the dynamo can sometimes survive a relatively strong imposed vertical flux - the dynamo can fight against its suppression.

As discussed by, e.g., Lacey \& Fall (1985) and Moss et al. (2000), the main mechanisms resulting in angular momentum transport with ensuing systematic radial flow in spiral galaxies are (in order of importance) the nonaxisymmetric gravitational field of a bar or spiral arms (Lubow et al. 1986), viscous and magnetic torques (Mestel 1999), and the infall of matter onto the disc (Pitts \& Tayler 1996). The magnitude of the radial velocity and its radial profile are uncertain; the plausible values range from 1 to $10 \mathrm{~km} \mathrm{~s}^{-1}$ at a distance of order $3-10 \mathrm{kpc}$ from the centre in a spiral galaxy such as the Milky Way.

We examine two basic galaxy models. One has a gas density distribution taken from Milky Way observations in $r>2$ kpc (see Sect. 3.2) but, in order to allow examination of a wider range of parameters we limited the central gas density in $r \leq 2 \mathrm{kpc}$ to unrealistically small values. These calculations are described in Sects. 5.1 and 5.2. In Sect. 6 we take a more realistic value of the central gas density, which is very strongly peaked in $r<2 \mathrm{kpc}$, and present results for a limited range of parameters applicable to the Milky Way. Thus readers who are only interested in applications to the Milky Way might consider omitting reading Sects. 5.1 and 5.2.

We emphasize that we consider a prescribed dipolar flux, trapped by the disc at the time of galaxy formation. In reality, a galaxy is likely to trap a mixed parity flux, but the quadrupolar component will merely act as a seed for the dynamo.

\section{The model}

\subsection{Basic equations}

We study a dynamo active disc embedded in a passive, conducting halo, and solve the standard $\alpha^{2} \omega$ dynamo equation in the disc,

$$
\frac{\partial \boldsymbol{B}}{\partial t}=\nabla \times(\boldsymbol{v} \times \boldsymbol{B}+\alpha \boldsymbol{B})-\nabla \times(\eta \nabla \times \boldsymbol{B}),
$$

but introduce an inwardly directed axisymmetric radial accretion flow and an axisymmetric vertical magnetic flux. The $\alpha$-effect and turbulent magnetic diffusion are specified via scalar fields $\alpha$ and $\eta$. We study axisymmetric solutions of Eq. (1) in the domain $-Z \leq z \leq Z, r_{\min } \leq r \leq R$, and define the aspect ratio of the domain as $\xi=Z / R$, where $r$ and $z$ are cylindrical polar coordinates. We choose

$\alpha= \begin{cases}\frac{\alpha_{0} \sin (\pi z / h)}{1+\boldsymbol{B}^{2} / 4 \pi k \rho v_{\mathrm{t}}^{2}}, & |z| \leq h, \\ 0, & |z|>h,\end{cases}$

defining the semi-thickness of the dynamo active disc, $h$, assumed to be independent of $r$ (of course, $h<Z$ ). Here $\alpha_{0}$ is the background (unquenched) magnitude of the $\alpha$ effect, the gas density is denoted $\rho$ (a function of $\boldsymbol{r}$ ), and $v_{\mathrm{t}}$ is the turbulent velocity. We also define the disc aspect ratio $\lambda=h / R$. We have thus introduced a conventional $\alpha$-quenching nonlinearity, with $k$ a constant of order unity (reflecting some of the uncertainty in our ideas about the reaction of the large scale magnetic field on to the turbulent motions, but ignoring ongoing controversies about the nature and effectiveness of the feedback of the dynamo 
generated fields on the $\alpha$-effect - see, e.g., Vainshtein \& Cattaneo 1992; Blackman \& Field 2000; Kleeorin et al. 2000; Brandenburg \& Subramanian 2000).

Since we will consider only axisymmetric solutions, we can write

$\boldsymbol{B}=B_{\phi} \widehat{\boldsymbol{\phi}}+\nabla \times\left(A_{\phi} \widehat{\boldsymbol{\phi}}\right)$,

where $B_{\phi}$ and $A_{\phi}$ are the azimuthal components of the magnetic field and vector potential, respectively. We also put

$\boldsymbol{v}=u(\boldsymbol{r}) \widehat{\boldsymbol{r}}+r \Omega(r) \widehat{\boldsymbol{\phi}}+\boldsymbol{u}_{\mathrm{dia}}$,

where $\boldsymbol{u}_{\text {dia }}=-\frac{1}{2} \nabla \eta$ allows for the turbulent diamagnetism (Zeldovich 1956; Roberts \& Soward 1975), $u$ is the prescribed radial inflow, and $\Omega$ is the galactic angular velocity.

For the magnetic diffusivity, we set

$\eta= \begin{cases}\eta_{0}, & |z| \leq h, \\ \eta_{0}\left[1+\left(\eta_{1}-1\right) \Theta(z)\right], & |z|>h,\end{cases}$

where $\eta_{0}$ is a constant,

$\Theta(z)=1-\exp \left[-\frac{(|z|-h)^{2}}{l_{\eta}^{2}}\right]$

is a smoothed step function and, rather arbitrarily, $l_{\eta}=$ $\frac{1}{2}(\xi-\lambda)$. Thus, the constant $\eta_{1}$ is the ratio of the diffusion coefficient high in the halo to that in the disc. We consider models with the turbulent magnetic diffusivity in the halo significantly larger than in the disc. This appears to be a reasonable assumption for a galactic disc surrounded by a hot, turbulent halo (Sokoloff \& Shukurov 1990; Poezd et al. 1993) as well as for an accretion disc corona supported by turbulent pressure (Ouyed \& Pudritz 1997). Anyway, the larger is the magnetic diffusivity outside the disc, the easier is the dragging of the vertical magnetic flux.

\subsection{A model for the Galaxy}

We take $\Omega$ from a model of the Milky Way (Burton \& Gordon 1978; see also p. 123 of Ruzmaikin et al. 1988), and show $V=r \Omega(r)$ in Fig. 1. The surface density of the interstellar gas $\sigma$ is taken from Sanders et al. (1984), and also shown in Fig. 1 for $r \geq 2 \mathrm{kpc}$. According to Sanders et al. (1984) (see also Dame 1992), the surface density of molecular hydrogen increases to $64 M_{\odot} \mathrm{pc}^{-2}$ at $r=1 \mathrm{kpc}$ and a few hundred $M_{\odot} \mathrm{pc}^{-2}$ inside that radius. In our calculations we use both a central density of $300 M_{\odot} \mathrm{pc}^{-2}$ and also a naive extrapolation of Fig. 1 to $5 M_{\odot} \mathrm{pc}^{-2}$ at $r=0$. The latter value is used to allow exploration of general properties of dynamos with inflow without the necessity of using a very high radial resolution. These distributions of $\rho$ and $r \Omega$ describe a rather old-fashioned model of the Milky Way, with the Solar galactocentric distance and rotation speed being $10 \mathrm{kpc}$ and $250 \mathrm{~km} \mathrm{~s}^{-1}$, rather than the currently accepted $8.5 \mathrm{kpc}$ and $220 \mathrm{~km} \mathrm{~s}^{-1}$, but we do not believe that this choice affects our conclusions.

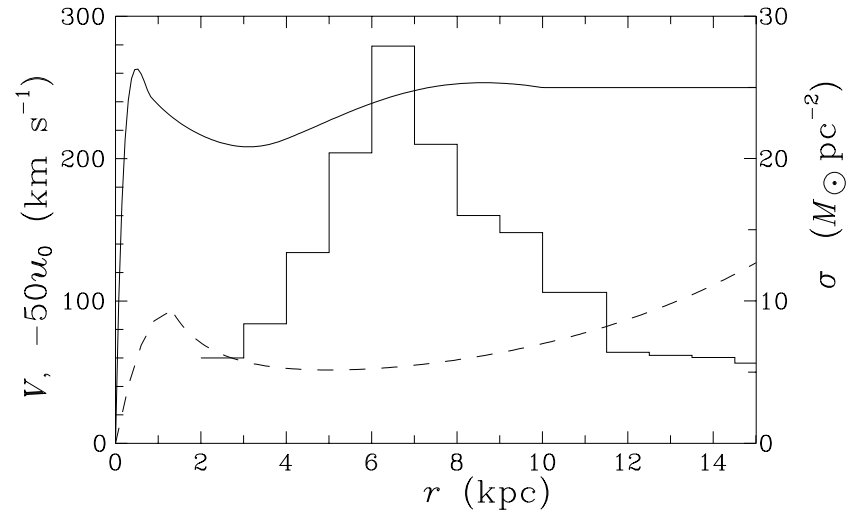

Fig. 1. The radial dependence of the Galactic rotation velocity $V=r \Omega$ (solid) (Burton \& Gordon 1978), the radial velocity in the disc midplane $u_{0}$ (multiplied by -50 ), Eq. (4) (broken), and gas surface density $\sigma$ at $r \geq 2 \mathrm{kpc}$ (Sanders et al. 1984) (histogram).

We neglected any possible $z$-dependence of $\Omega$ outside of the disc. This is rather uncertain, and would not dramatically affect the computed fields, given that the inflow and dynamo action are confined to the disc region, and so also is the bulk of the magnetic field. We neglect the possibility of any dynamo action in the halo (Sokoloff \& Shukurov 1990; Brandenburg et al. 1992, 1993).

With $\eta$ growing with $z$, turbulent magnetism, resulting in effective magnetic field transport at a velocity $\boldsymbol{u}_{\text {dia }}$, compresses magnetic field to the disc (Brandenburg et al. 1992; Gabov et al. 1996). However, this effect is relatively unimportant.

Moss et al. (2000) discuss a crude but plausible model for the radial profile of a radial flow in the disc plane of a spiral galaxy, valid over all but the innermost part of the disc plane,

$u_{0}(r)=-1.4 \frac{\mathrm{km}}{\mathrm{s}} \mathrm{e}^{\left(r-R_{\odot}\right) / r_{0}}\left(\frac{r}{R_{\odot}}\right)^{-1}\left(\frac{\dot{M}}{1 M_{\odot} \mathrm{yr}^{-1}}\right)$,

where they put $r_{0}=5 \mathrm{kpc}$. With the density profile shown in Fig. 1, the resulting mass accretion rate $\dot{M} \simeq 2 \pi r \sigma u_{0} \approx$ $1 M_{\odot} \mathrm{yr}^{-1}$ is independent of radius within 20 per cent in $5<r<15 \mathrm{kpc}$, and decreases at smaller $r$ (mimicking a mass sink due to star formation in the galactic molecular ring). In order to avoid large flow velocities in the inner part of the disc, where observational data are poor and the situation uncertain, we caused $u_{0}$ to go smoothly to zero between $r=r_{u}=1.5 \mathrm{kpc}$ and $r=0$. Then if $u_{0}=$ $-1 \mathrm{~km} \mathrm{~s}^{-1}$ at the Solar position, we have $u_{0} \approx-0.6 \mathrm{~km} \mathrm{~s}^{-1}$ at $r=300 \mathrm{pc}$, which corresponds to $\dot{M} \approx 0.3 M_{\odot} \mathrm{yr}^{-1}$ at that radius (for $\sigma=300 M_{\odot} \mathrm{pc}^{-2}$ ), as given by Morris \& Serabyn (1996). We also explored the effect of reduction in the value of the truncation radius $r_{u}$ (see Sect. 6.2).

The resulting radial velocity profile $u_{0}$ is shown in Fig. 1. We also define a function

$f_{u}(z)= \begin{cases}1, & |z| \leq h \\ \exp \left[-(|z| / h-1)^{2}\right], & |z|>h\end{cases}$ 
and put $u(r, z)=u_{0}(r) f_{u}(z)$, ensuring that the inflow is essentially zero away from the disc.

We consider a range of models with various values of $u_{0}\left(R_{\odot}\right)$ in Eq. (4) by replacing the factor 1.4 by $0.8 U_{0}$ and varying $U_{0} ; u_{0}\left(R_{\odot}\right) \simeq-1 \mathrm{~km} \mathrm{~s}^{-1}$ seems to be the most plausible value.

\subsection{Introduction of dimensionless variables}

Equation (1) is nondimensionalized in terms of a length $R$ and a time $h^{2} / \eta_{0}$. We measure angular velocity, radial velocity and $\alpha$ in units of their typical values $\Omega_{0}, U_{0}$ and $\alpha_{0}$ respectively, and $\boldsymbol{B}$ in units of $B_{0}=\left(4 \pi k \rho_{0} v_{\mathrm{t}}^{2}\right)^{1 / 2}$, an equipartition value with respect to the turbulent kinetic energy. The choice $\rho_{0}=5 \times 10^{-24} \mathrm{~g} \mathrm{~cm}^{-3}$ corresponds to the maximum surface density given by Sanders et al. (1984) outside the central region, which is about $28 M_{\odot} \mathrm{pc}^{-2}$, with the effective scale height of the neutral gas of $200 \mathrm{pc}$ taken to be independent of $r$. The gas density $\rho$ was taken to be independent of $z$ in $|z| \leq h$; its value in $|z|>h$ is irrelevant, as $\alpha=0$ there, and $\rho$ only appears in the $\alpha$-quenching term. We took $\Omega_{0}=25 \mathrm{~km} \mathrm{~s}^{-1} \mathrm{kpc}^{-1}$.

The poloidal and toroidal parts of Eq. (1) become respectively

$$
\begin{aligned}
\frac{\partial A}{\partial t}=( & \left.\lambda R_{\alpha} \alpha \boldsymbol{B}+\lambda R_{u} u \widehat{\boldsymbol{r}} \times \boldsymbol{B}+\lambda^{2} \boldsymbol{u}_{\mathrm{dia}} \times \boldsymbol{B}\right) \cdot \widehat{\boldsymbol{\phi}} \\
& +\lambda^{2} \eta \mathcal{D}^{2} A, \\
\frac{\partial B}{\partial t}=\nabla & \times\left(\lambda R_{\alpha} \alpha \boldsymbol{B}+\lambda R_{u} u \widehat{\boldsymbol{r}} \times \boldsymbol{B}+R_{\omega} \Omega r \widehat{\boldsymbol{\phi}} \times \boldsymbol{B}\right. \\
& \left.+\lambda^{2} \boldsymbol{u}_{\mathrm{dia}} \times \boldsymbol{B}\right) \cdot \widehat{\boldsymbol{\phi}}+\lambda^{2} \eta \mathcal{D}^{2} B,
\end{aligned}
$$

where $A$ and $B$ are the dimensionless forms of $A_{\phi}$ and $B_{\phi}$, and

$\mathcal{D}^{2}=\frac{\partial^{2}}{\partial r^{2}}+\frac{1}{r} \frac{\partial}{\partial r}-\frac{1}{r^{2}}+\frac{\partial^{2}}{\partial z^{2}}$.

The radial velocity in Eqs. (5) and (6) is measured in units of $U_{0}$, giving

$u_{0}=-0.05(r / R)^{-1} \exp \left(r / r_{0}\right)$.

In these equations, and below, we refer to dimensionless quantities, unless otherwise stated. Thus we have the dimensionless parameters

$R_{\alpha}=\frac{\alpha_{0} h}{\eta_{0}}, \quad R_{\omega}=\frac{\Omega_{0} h^{2}}{\eta_{0}}, \quad R_{u}=\frac{U_{0} h}{\eta_{0}}$.

We chose $R=15 \mathrm{kpc}, Z=5 \mathrm{kpc}, h=500 \mathrm{pc}$ (the scale height of the ionized gas, distinct from that of the neutral gas), $\eta_{1}=30$, and considered two values of the disc magnetic diffusivity, $\eta_{0}=10^{26}$ and $3 \times 10^{26} \mathrm{~cm}^{2} \mathrm{~s}^{-1}$. The former (more conventional) value of $\eta_{0}$ determines $R_{\omega}=18.75$, the latter $R_{\omega}=6.25$, and both suggest that if $\alpha_{0}$ is of order $1 \mathrm{~km} \mathrm{~s}^{-1}$, then values of $R_{\alpha}$ of order unity are plausible; below we take $R_{\alpha}=0.5$ and 2 , respectively, both somewhat supercritical values. With $\eta_{0}=10^{26}$ and $3 \times 10^{26} \mathrm{~cm}^{2} \mathrm{~s}^{-1}$, values of the radial magnetic Reynolds number $R_{u}=3$ and 1 , respectively, correspond to inward velocities of about $u_{0}=-1 \mathrm{~km} \mathrm{~s}^{-1}$ at the solar radius of $10 \mathrm{kpc}$. With this value of $u_{0}\left(R_{\odot}\right)$, the unit radial speed is $U_{0} \approx 1.8 \mathrm{~km} \mathrm{~s}^{-1}$. If $v_{\mathrm{t}}=10 \mathrm{~km} \mathrm{~s}^{-1}$ (at all radii), the unit of magnetic field is $B_{0}=8 k^{1 / 2} \mu \mathrm{G}$, and the unit of magnetic energy is $\frac{1}{2} B_{0}^{2} R^{2} Z$.

\subsection{Boundary conditions}

At the boundaries $z= \pm \xi$, we used the boundary conditions $\partial B / \partial z=0, B_{r}=-\partial A / \partial z=0$. If the inner radius of the computational domain is at $r_{\min }=0$, then regularity demands $A=B=0$ at $r=r_{\min }$. For the many of our computations we took, for numerical convenience, $r_{\min }=0.02$, and applied boundary conditions on $A$ and $B$ that were consistent with the analytical behaviour as $r \rightarrow 0$; most importantly $\partial B_{z} / \partial r=0$. We also used $r_{\min }=0$ for some computations - comparison between solutions with these values showed good agreement. The boundary condition at $r=1$ is $B=0$, and the initial field is a small amplitude seed of mixed parity.

We set $A=\frac{1}{2} \mathcal{B}$ at all times on the outermost radial grid line $r=1$ with $\mathcal{B}=$ const, and solve the difference equations resulting from Eqs. (5) and (6) in the region $r_{\min } \leq r \leq 1$. With $B_{z}=r^{-1} \partial(r A) / \partial r$, this specifies a constant vertical magnetic flux through the disc midplane $z=0$, given by $F=2 \pi \int_{0}^{1} B_{z} r \mathrm{~d} r \equiv \pi \mathcal{B}$ in dimensionless units. This prescription means that at time $t=0$, when $A$ is negligible at the inner grid lines, there is formally a discontinuity in $A$, and so an infinity of $B_{z}$, near the boundary $r=1$. However this is immediately smoothed by diffusion, and the field is smooth after a few time steps. Our interest is mainly with stationary magnetic configurations (either steady or oscillatory) established at later stages of evolution, especially as initial conditions are physically uncertain. We confirmed, by performing a trial integration with the boundary condition that $B_{z}$ was uniform on the boundaries $z= \pm \xi$, for a prescribed value of the flux, that our computational procedure led to the unique solution.

Thus the interpretation of $\mathcal{B}$ is that it is the uniform field in the $z$-direction that would have the same total flux through the disc plane $z=0, r \leq 1$ as the model considered.

Equations (5) and (6) are discretized on a grid with $N_{r}$ points uniformly distributed over the range $r_{\text {min }} \leq r \leq 1$ and $N_{z}$ points distributed over $-\xi \leq z \leq \xi$. The spatial discretization is second order accurate in space, and a second order Runge-Kutta method was used to advance the solution in time. Our standard resolution was $N_{r}=51$, $N_{z}=201$, but we used $N_{r}=401$ in the models with large central density, $\sigma(0)=300 M_{\odot} \mathrm{pc}^{-2}$.

\subsection{Diagnostic quantities}

Four key parameters are used to quantify the properties of our stationary solutions, denoted by $E, P, P_{\mathrm{i}}$ and $\varphi$. $E$ is defined to be the total magnetic field energy in the computational domain. 
The global parity $P$ is defined as $P=\left(E_{\text {even }}-\right.$ $\left.E_{\text {odd }}\right) / E$, where $E_{\text {even }}$ and $E_{\text {odd }}$ are the global energies of the parts of the field possessing respectively quadrupolelike and dipole-like symmetry with respect to $z=0$ (cf. Brandenburg et al. 1992). Thus a purely quadrupole-like field has $P=+1$, and $P=-1$ for a dipole-like field. We similarly define $P_{\mathrm{i}}$, the field parity in the region interior to $r_{\mathrm{i}}=0.02$ (300 pc in dimensional units). We monitor $P_{\mathrm{i}}$ to allow for the possibility that dipolar magnetic field can dominate in the centre of the disc (with a predominantly quadrupolar global parity), as possibly observed in the Milky Way. As the dominant toroidal component of the magnetic field is strongly concentrated to the disc region, values of $E, P$ and $P_{\mathrm{i}}$ calculated for the disc region will be close to the values calculated for the complete disc+halo system.

The vertical magnetic flux within radius $r$ is $F(r)=$ $2 \pi \int_{0}^{r} B_{z} r \mathrm{~d} r=2 \pi A(r) r$, whereas the total magnetic flux through the disc midplane is $F=F(1)=\pi \mathcal{B}$. Then $F_{\mathrm{i}}=F(0.02)$ is the flux within radius $r=0.02$ (arising necessarily from the odd-parity part of the field), and the ratio $\varphi=F_{\mathrm{i}} / F$ is the fourth descriptor of our results. Note that for a strictly uniform field in the $z$-direction we have $A(r) \propto r$, and $\varphi=r_{\mathrm{i}}^{2}=4 \times 10^{-4}$.

To summarise, our results are presented in terms of the total magnetic energy $E$, the global magnetic parity $P$, the parity $P_{i}$ of the field in the inner $300 \mathrm{pc}$ of the disc, and $\varphi$, the relative vertical magnetic flux through the inner 300 pc. The average dimensionless vertical magnetic field within the inner $300 \mathrm{pc}$ can be obtained as $\bar{B}_{z, \mathrm{c}}=$ $2.5 \times 10^{3} \mathcal{B} \varphi$.

\section{The reference states}

In this section we briefly describe two basic states of the system considered - firstly, dragging of an imposed vertical flux without any dynamo action and, secondly, a disc dynamo without any imposed flux or radial flow. These states provide references against which the relative importance of dynamo action and advection in the composite model can be assessed. For the dynamo model described in Sect. 4.2 we take the lower of the values discussed for the central gas density, $\sigma(0)=5 M_{\odot} \mathrm{pc}^{-2}$. The corresponding dynamo model for the high central density case, $\sigma(0)=300 M_{\odot} \mathrm{pc}^{-2}$ is described in Sect. 6.1. The gas density does not enter the purely advective-diffusive calculation discussed in Sect. 4.1.

\subsection{Magnetic flux captured by a dynamo inactive disc}

We start by considering the advection of imposed vertical flux by a radial inflow in a differentially rotating disc in the absence of dynamo action. Without the $\alpha$-effect in Eq. (1), the Eqs. (5) and (6) for $A$ and $B$ decouple and their solutions as $t \rightarrow \infty$ can differ from zero only because $\mathcal{B} \neq 0$. So it is clear that, for given values of $R_{u}$ and $R_{\omega}$, solutions will scale with the single parameter $\mathcal{B}$, with magnetic energy $E_{\mathrm{adv}} \propto \mathcal{B}^{2}$. Equilibrium is determined solely

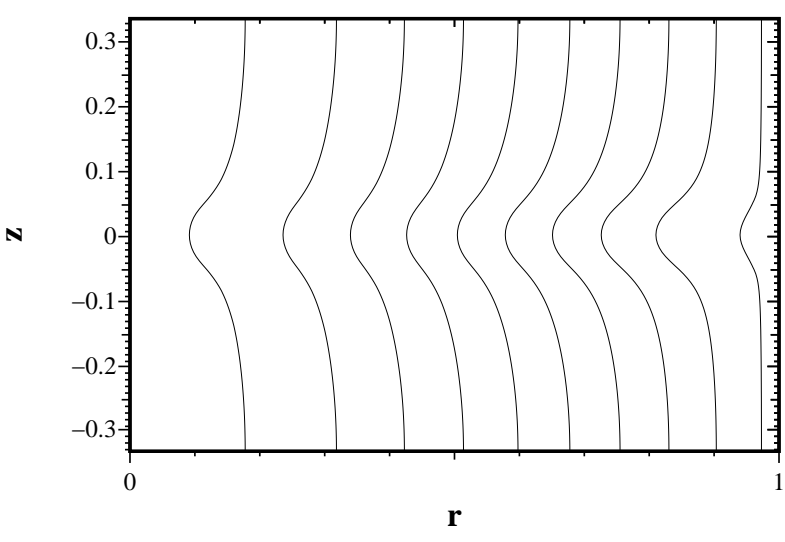

(a)

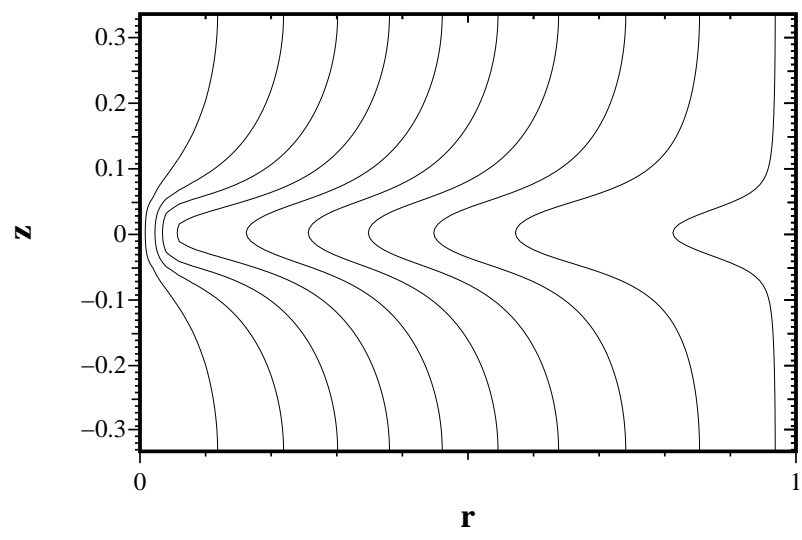

(b)

Fig. 2. The poloidal field lines (equally spaced in poloidal magnetic flux $r A$ ) in a steady state, for the kinematic advection calculation of Sect. 4.1 with $R_{\omega}=18.75, \eta_{0}=10^{26} \mathrm{~cm}^{2} \mathrm{~s}^{-1}$ $R_{\alpha}=0$, and a) $R_{u}=1.5$, and b) $R_{u}=6$.

by a balance of advection and diffusion, and the toroidal field $B$ is parasitic on $A$, via twisting of the poloidal field lines by the differential rotation and vertical shear in $u$. The field parity is odd, $P=-1$, as this is the parity of the imposed external field.

We show in Fig. 2 the poloidal field lines for $\eta_{0}=$ $10^{26} \mathrm{~cm}^{2} \mathrm{~s}^{-1}, R_{\omega}=18.75$, and $R_{u}=1.5$ and 6 . The effects of increasing the magnetic Reynolds number of the radial flow are clearly seen: field advection is markedly stronger when $R_{u}$ is larger.

\subsection{Disc dynamo without inflow}

When $R_{\alpha}$ is non-zero, but $R_{u}=0$ and $\mathcal{B}=0$, we have a standard disc dynamo. For reference, we show the field structure of a nonlinear solution with our standard parameters $R_{\alpha}=0.5, R_{\omega}=18.75, \eta_{0}=10^{26} \mathrm{~cm}^{2} \mathrm{~s}^{-1}$ in Fig. 3. The field parity is, as expected, quadrupolar $(P=+1)$. The toroidal field is strongly concentrated in the disc, $|z| \leq 0.0333$, where the $\alpha$-effect is non-zero, but the poloidal field pervades the halo. In this case we set 


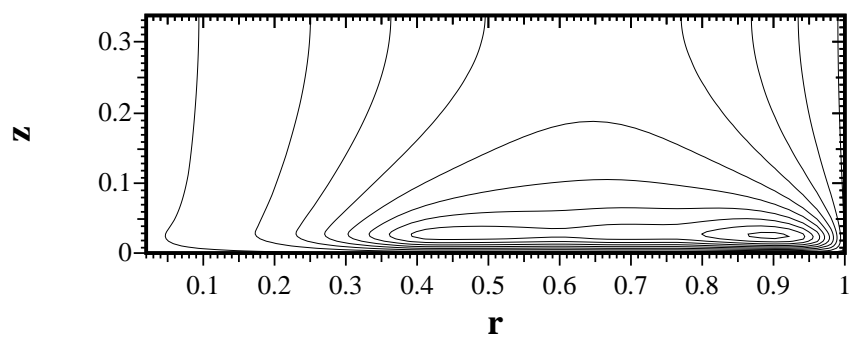

(a)

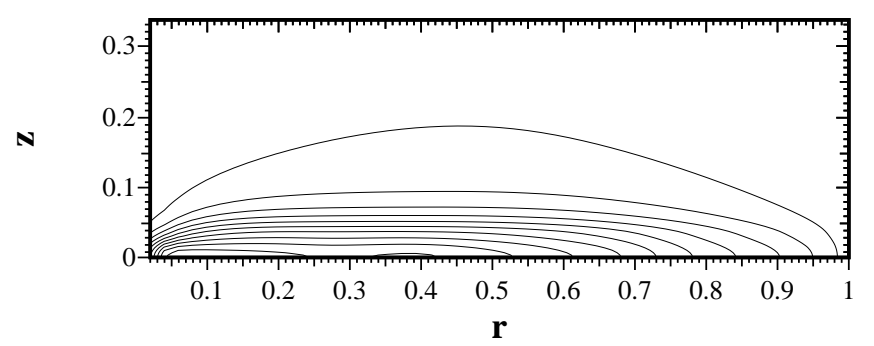

(b)

Fig. 3. The poloidal field lines a) and contours of toroidal field b), equally spaced in poloidal flux and toroidal field strength respectively, for the pure dynamo calculation of Sect. 4.2 with $R_{u}=0, R_{\omega}=18.75, \eta_{0}=10^{26} \mathrm{~cm}^{2} \mathrm{~s}^{-1}$ and $R_{\alpha}=0.5$. The field has an even (quadrupolar, $P=+1$ ) structure with respect to the midplane $(z=0)$, so only the upper half space is shown.

$\mathcal{B}=0$ but, in the absence of an inflow, the value of $\mathcal{B}$ is almost irrelevant.

\section{Dynamos with inflow; field advection and semi-dynamo action}

In this section we discuss axisymmetric steady state magnetic fields in a dynamo active disc where trapped vertical magnetic flux is dragged in from large radii by an axially symmetric flow. We explore ranges of $R_{u}$ and $\mathcal{B}$, the two less certain parameters of the model. The importance of the external magnetic field is also sensitive to the magnetic diffusivity $\eta$ in the disc - the dragging is more efficient when the magnetic field is more strongly coupled to the flow, i.e. when $\eta$ is smaller for a given inflow speed. We consider two values of $\eta$, one being a standard value for galactic discs, and the other a factor of three larger.

We first consider the "low" value for the central density, $\sigma=5 M_{\odot} \mathrm{pc}^{-2}$, in order to allow the use of a lower radial resolution. In Sect. 5.1 results from a basic model, where we choose a single set of typical Galactic parameters, are given. Then we go on to explore how the results are affected by changes in a few key parameters. Results for our "standard" magnetic diffusivity are presented in Sect. 5.1 and those using an enhanced diffusivity are in Sect. 5.2.

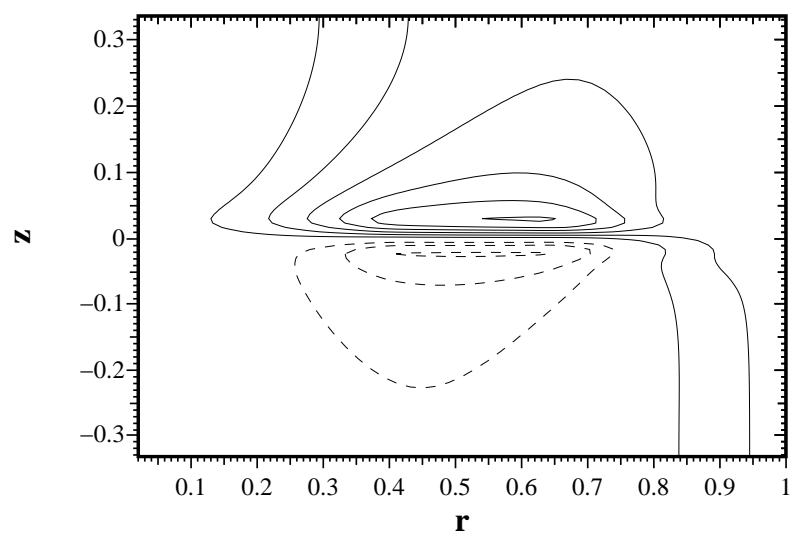

(a)

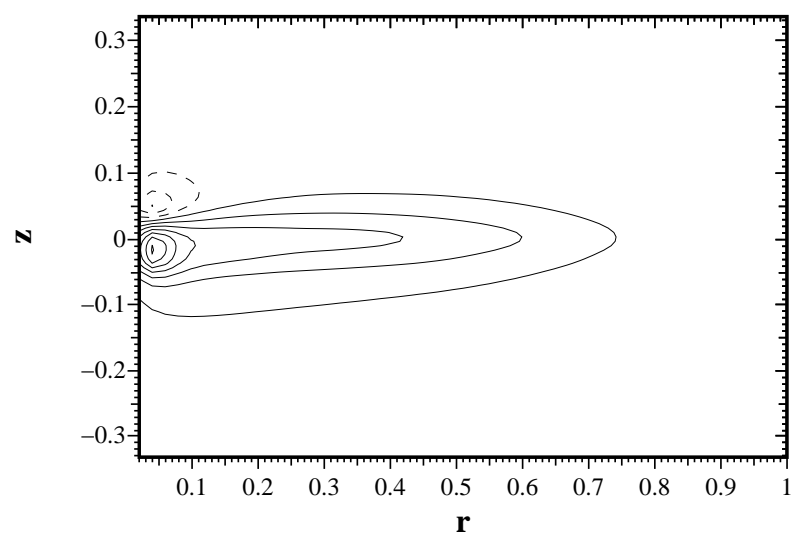

(b)

Fig. 4. The poloidal field lines a) and the contours of toroidal field $\mathbf{b}$ ), both equally spaced, for the calculation of Sect. 5.1 with $R_{u}=3, R_{\omega}=18.75, \eta_{0}=10^{26} \mathrm{~cm}^{2} \mathrm{~s}^{-1}, R_{\alpha}=0.5, \mathcal{B}=$ $10^{-3}$. Continuous and broken contours refer to positive and negative values respectively.

\subsection{Standard magnetic diffusivity: $\eta_{0}=10^{26} \mathrm{~cm}^{2} \mathrm{~s}^{-1}$}

We begin by describing a typical nonlinear model with $\eta_{0}=10^{26} \mathrm{~cm}^{2} \mathrm{~s}^{-1}$, choosing parameters $\mathcal{B}=10^{-3}$ (corresponding to a mean vertical field of $8 \times 10^{-9} \mathrm{k}^{1 / 2} \mathrm{G}$ in dimensional units), $R_{u}=3, R_{\alpha}=0.5$ and $R_{\omega}=18.75$.

We display in Fig. 4 the steady state magnetic field structure found in this case. There are clear deviations from pure parity, either odd or even. The global parity parameter is $P=0.85$ indicating an almost quadrupolar parity, whereas in the inner $2 \%$ by radius $P_{\mathrm{i}}=0.02$. A small value of $\left|P_{\mathrm{i}}\right|$ means that the magnetic field is relatively small either above or below the disc, in this case in $z>0$ - see Fig. 4b.

Our results are summarized in Table 1. The fields evolve to steady configurations, except with the smaller $\mathcal{B}$ values with $R_{u}=6$, when the dynamo is barely excited when $\mathcal{B}=0$ and oscillates for $\mathcal{B}=0,10^{-3}$ and $10^{-4}$. 
Table 1. Summary of results for $\eta_{0}=10^{26} \mathrm{~cm}^{2} \mathrm{~s}^{-1}$, the central gas surface density $\sigma(0)=5 M_{\odot} \mathrm{pc}^{-2}$ and $R_{u}=0,1.5,3$, and 6 . The two parity values given are respectively the global parity $P$ and that in the inner $2 \%$ by radius, $P_{\mathrm{i}}$. The values of $E$ for $\mathcal{B}=0$ are those for the pure dynamo, $E_{\text {dyn }}$. The magnetic energy obtained in the absence of dynamo action, $E_{\text {adv }}$, scales with $\mathcal{B}^{2}$, but is given explicitly for convenience. $\varphi=F_{\mathrm{i}} / F$ is the fraction of the magnetic flux within radius $r=0.02$, and $\bar{B}_{z, \mathrm{c}}$ is the dimensionless mean vertical field through $z=0$ within radius $r=r_{i}=0.02$ (corresponding to the inner $300 \mathrm{pc}$ in radius). There are no entries for $\varphi$ when $\mathcal{B}=0$, as there is zero flux through the midplane for a purely even parity field. Where a range of values are given, the solutions are oscillatory, and the values are maxima and minima. Note that the flux parameter $\mathcal{B}$ is the strength of the (dimensionless) uniform vertical field that would have the same flux through the midplane as the corresponding model. For reference, when $R_{u}=0, \mathcal{B}=0$, we find $\left(P, P_{i}, E\right)=(+1,+1,0.64)$.

\begin{tabular}{|c|c|c|c|c|c|c|}
\hline $\mathcal{B}$ & 1 & $10^{-1}$ & $10^{-2}$ & $10^{-3}$ & $10^{-4}$ & 0 \\
\hline \multicolumn{7}{|c|}{$R_{u}=1.5$} \\
\hline$P$ & -1 & -0.994 & 0.17 & 0.98 & 0.9998 & +1 \\
\hline$P_{\mathrm{i}}$ & -1 & -0.9999 & -0.82 & 0.82 & 0.997 & +1 \\
\hline$E$ & $1.13 \times 10^{3}$ & 1.68 & 0.80 & 0.48 & 0.48 & 0.48 \\
\hline$E_{\mathrm{adv}}$ & $1.16 \times 10^{3}$ & $1.16 \times 10^{1}$ & $1.16 \times 10^{-1}$ & $1.16 \times 10^{-3}$ & $1.16 \times 10^{-5}$ & 0 \\
\hline$\varphi$ & $4.5 \times 10^{-3}$ & $2.5 \times 10^{-3}$ & $5.7 \times 10^{-3}$ & $1.1 \times 10^{-2}$ & $1.2 \times 10^{-2}$ & - \\
\hline $\bar{B}_{z, \mathrm{c}}$ & 11.3 & 0.63 & 0.14 & 0.028 & 0.0030 & 0 \\
\hline \multicolumn{7}{|c|}{$R_{u}=3$} \\
\hline$P$ & -1 & -1 & -0.55 & 0.85 & 0.998 & +1 \\
\hline$P_{\mathrm{i}}$ & -1 & -1 & -0.98 & 0.02 & 0.98 & +1 \\
\hline$E$ & $7.56 \times 10^{3}$ & 48.4 & 1.08 & 0.32 & 0.30 & 0.32 \\
\hline$E_{\mathrm{adv}}$ & $7.60 \times 10^{3}$ & $7.60 \times 10^{1}$ & $7.60 \times 10^{-1}$ & $7.60 \times 10^{-3}$ & $7.60 \times 10^{-5}$ & 0 \\
\hline$\varphi$ & $2.1 \times 10^{-2}$ & $2.1 \times 10^{-2}$ & $2.2 \times 10^{-2}$ & $4.5 \times 10^{-2}$ & $4.5 \times 10^{-2}$ & - \\
\hline $\bar{B}_{z, \mathrm{c}}$ & 52.5 & 5.3 & 0.55 & 0.11 & 0.011 & 0 \\
\hline \multicolumn{7}{|c|}{$R_{u}=6$} \\
\hline$P$ & -1 & -1 & -1 & $-0.35 \ldots 0.72$ & $0.966 \ldots 0.997$ & +1 \\
\hline$P_{\mathrm{i}}$ & -1 & -1 & -1 & $-0.93 \ldots 0.78$ & $0.45 \ldots 0.998$ & +1 \\
\hline$E$ & $4.08 \times 10^{4}$ & $3.48 \times 10^{2}$ & $1.41 \times 10^{-1}$ & $(1.42 \ldots 14) \times 10^{-3}$ & $(5.8 \ldots 128) \times 10^{-4}$ & $(5.6 \ldots 128) \times 10^{-4}$ \\
\hline$E_{\mathrm{adv}}$ & $4.10 \times 10^{4}$ & $4.10 \times 10^{2}$ & 4.10 & $4.10 \times 10^{-2}$ & $4.10 \times 10^{-4}$ & 0 \\
\hline$\varphi$ & 0.125 & 0.117 & 0.021 & $0.0067 \ldots 0.030$ & $0.0051 \ldots 0.030$ & - \\
\hline $\bar{B}_{z, \mathrm{c}}$ & 312.0 & 29.3 & 0.53 & $0.017 \ldots 0.075$ & $0.013 \ldots 0.0075$ & 0 \\
\hline
\end{tabular}

\subsubsection{Field parity and strength}

Two trends are apparent. As illustrated in Fig. 5, for given $R_{u}$, increasing $\mathcal{B}$ causes the parity to become increasingly negative - i.e. more dipole-like. However, this effect is uniform neither in radius, nor between the poloidal and toroidal field components. We show in Fig. 6 the evolution of the parity of the poloidal and toroidal parts of the field for the calculation illustrated in Fig. 4. Of course, as the energy in the toroidal part of the field is much greater than that in the poloidal part, the parity of the toroidal field dominates the global parity. For a given $\mathcal{B}$ and $R_{u}$, the magnetic field in the inner disc $r<0.02$ is more dipole-like than in the rest of the disc as a stationary field configuration is approached (i.e., $P_{\mathrm{i}}$ is systematically smaller than $P)$. This also applies to the lower values attained by $P$ and $P_{\mathrm{i}}$ in oscillatory solutions.

Secondly, increasing $R_{u}$ decreases the dynamo efficiency, both in the linear and nonlinear regimes. This is masked to some extent when $\mathcal{B} \neq 0$ by the increasingly efficient inward advection of the vertical field. When $\mathcal{B}=0$, we find that the total dynamo-generated field energy decreases as $R_{u}$ increases: the trend is consistent with the findings of Moss et al. (2000) who, however, mostly considered linear solutions. We note that the magnetic energy (mean taken when oscillatory) of these two-dimensional solutions with $\mathcal{B}=0$ varies approximately linearly with $R_{u}$ as

$E_{\mathrm{dyn}} \approx E_{0}\left(1-R_{u} / 6\right)$,

with $E_{0}=0.64$ for $\eta=10^{26} \mathrm{~cm} \mathrm{~s}^{-1}, 0 \leq R_{u} \leq 6$ (see Table 1) and $E_{0}=0.90$ for $\eta=3 \times 10^{26} \mathrm{~cm} \mathrm{~s}^{-1}, 0 \leq R_{u} \leq$ 5 (see Table 2 ; we also note that $E_{\mathrm{dyn}}=0.46$ for $R_{u}=3$ consistent with this fit).

The result that advection inhibits dynamo action appears quite general; Moss et al. (2000) produced an analytic argument, and our numerical results arguably apply to an unfavourable case, as the advection tends to sweep the field towards the central regions where the local growth rate is largest. 


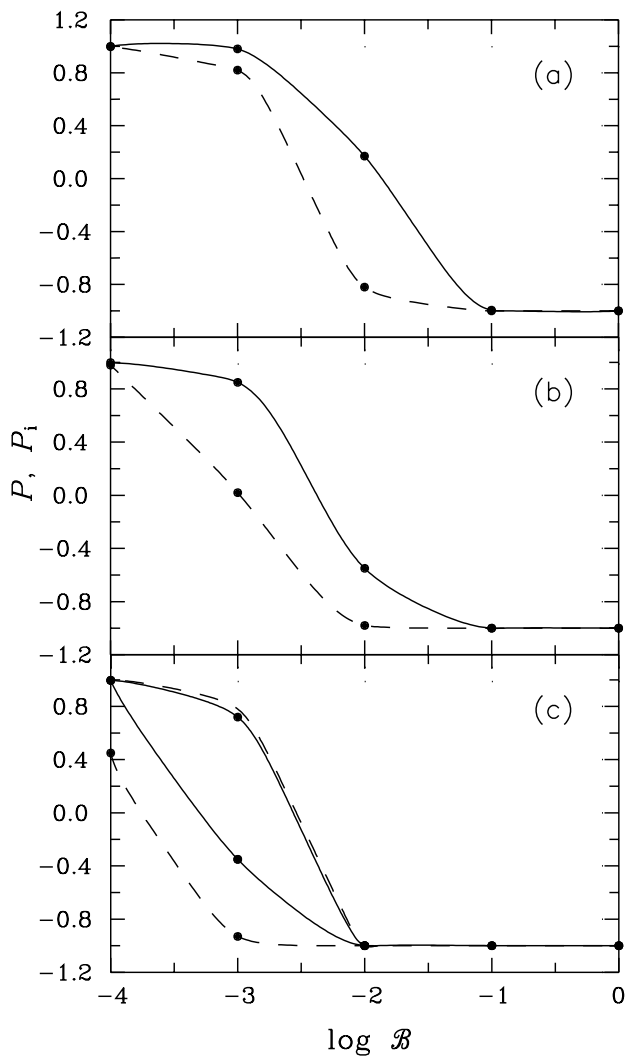

Fig. 5. The field parity in the whole disc, $P$ (solid), and in the inner region of the disc $r<0.02, P_{\mathrm{i}}$ (broken), versus the vertical flux parameter $\mathcal{B}$ for the calculation of Sect. 5.1 .1 with $R_{u}=1.5, R_{\omega}=18.75, \eta_{0}=10^{26} \mathrm{~cm}^{2} \mathrm{~s}^{-1}$, and $R_{\alpha}=0.5$. Panels a), b), c) are for the cases $R_{\omega}=1.5,3,6$ respectively. In c), the range of variation of the oscillatory solutions is indicated when $\mathcal{B}<10^{-2}$. The field approaches purely odd parity, i.e. $P \rightarrow-1$, as $\mathcal{B}$ increases, but the field near the centre of the disc has a systematically smaller value of $P$ (i.e., is more dipole-like) than that in the disc as a whole, and needs a weaker external field $\mathcal{B} \simeq 10^{-2}$ to become approximately dipolar.

The dynamo and the inward advection of the vertical field combine in a non-additive manner. In Fig. 7 (see also Table 1), we show the magnetic energy in a stationary state, together with that resulting from pure advection $\left(R_{\alpha}=0\right)$, and pure dynamo action $(\mathcal{B}=0)$. The total hybrid field energy $E$ consistently exceeds $E_{\mathrm{adv}}$ and is close to $E_{\text {dyn }}$ when $\mathcal{B} \leq 10^{-2}$, whereas $E<E_{\text {adv }}$ for $\mathcal{B}=1$; we deduce that $E \rightarrow E_{\text {adv }}$ as $\mathcal{B} \rightarrow \infty$.

The behaviour of the system when $R_{u}=6$ differs somewhat from that for the smaller values of $R_{u}$. Now an oscillatory dynamo is excited when $\mathcal{B}=0$ (see Fig. 8). The stationary state has relatively small energy and $E_{\mathrm{adv}}$ exceeds $E_{\text {dyn }}$ for $\mathcal{B} \gtrsim 10^{-3}$. Nevertheless, dynamo action plays a significant role; in the range $10^{-3} \leq \mathcal{B} \lesssim 1$ dynamo action again reduces the magnetic energy of the system below that of the purely advected field, so that $E<E_{\mathrm{adv}}$. This case shows some of the features of the model with $\eta_{0}=3 \times 10^{26} \mathrm{~cm}^{2} \mathrm{~s}^{-1}$ and $R_{u}=10$ discussed in Sect. 5.2.

The oscillatory behaviour of the solutions at large $R_{u}$ appears in the linear solutions with $\mathcal{B}=0$ also. However

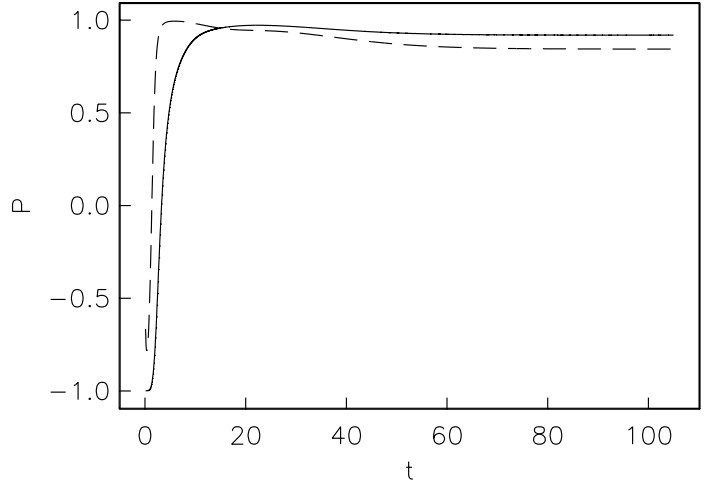

Fig. 6. The parity of the poloidal field (solid) and toroidal field (broken) in the whole computational domain as a function of time for the calculation illustrated in Fig. 4. (Time is measured in units of $7.5 \times 10^{8} \mathrm{yr}$ ).

the approximate linear solution of Eqs. (5) and (6) developed by Moss et al. (2000) in a one-dimensional approximation (retaining only $z$-derivatives, see their Eq. (A1)) remains non-oscillatory at any $R_{u}$, and this is confirmed by their numerical solutions based on the "no- $z$ " approximation. Thus the oscillations appear to arise from the explicit fully two-dimensional structure of the model. The period of these oscillations is of the order of the galactic age, so they appear to be of little importance for galaxies, but their origin in a broad dynamo theory context deserves further analysis. We have been unable to elucidate further either analytically or physically the mechanism driving these oscillations.

\subsubsection{Field concentration towards the disc centre}

Another notable feature of the stationary states is that the fraction of the vertical magnetic flux stored in the central part of the disc, $r<0.02$, exhibits a non-trivial dependence on both $R_{u}$ and $\mathcal{B}$. Without dynamo action, $\varphi$, the fraction of the vertical magnetic flux stored within $r=0.02$, increases with $R_{u}$. These values of $\varphi$ are identical to those shown in Table 1 for $\mathcal{B}=1$, i.e. the solutions with large $\mathcal{B}$ are close to those for pure advection. Larger values of $\varphi$ indicate stronger concentration of $B_{z}$ towards the disc axis. Note that $\varphi=4 \times 10^{-4}$ for a uniform vertical field. For dynamos without external vertical field (the last column in Table 1), $\varphi$ is not defined, because the field has quadrupolar parity and hence has no flux through the midplane $z=0$.

As illustrated in Table 1 , for $R_{u}=1.5$ (moderate inflow), $\varphi$ unexpectedly grows as $\mathcal{B}$ decreases below 0.1 . This growth is less pronounced for a stronger inflow, $R_{u}=3$. The trend reverses as the inflow becomes very strong, with $\varphi$ decreasing as $\mathcal{B}$ decreases. Thus, a larger trapped vertical flux results in a weaker central concentration of the vertical magnetic flux in the central part of a dynamo active disc provided the inflow in not too strong.

For a fixed $\mathcal{B}$, the field is more strongly concentrated to the centre when the inflow is stronger - a natural 
behaviour. This behaviour occurs for $\mathcal{B} \gtrsim 10^{-2}$ at all values of $R_{u}$ explored and for $R_{u}<6$ for all values of $\mathcal{B}$. Oscillatory solutions arising for $R_{u}=6$ behave differently having smaller maximum values of $\varphi$ than steady solutions with the same $\mathcal{B}$ but smaller $R_{u}$.

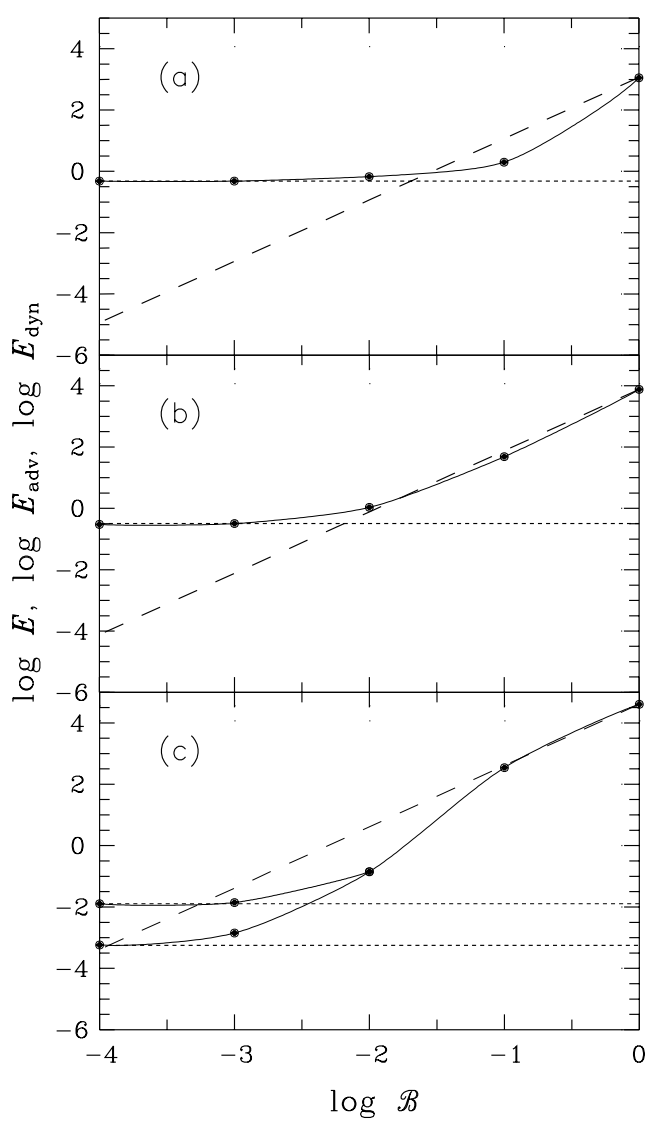

Fig. 7. The total field energy $E$, versus the flux parameter $\mathcal{B}$ for the calculation of Sect. 5.1.1. $E$ is shown as a solid line for $\mathbf{a}$ ) $\left.R_{u}=1.5, \mathbf{b}\right) R_{u}=3$ and c) $R_{u}=6$. The other parameters are $R_{\omega}=18.75, \eta_{0}=10^{26} \mathrm{~cm}^{2} \mathrm{~s}^{-1}$, and $R_{\alpha}=0.5$. The solution for an advected field with no dynamo action, i.e. $R_{\alpha}=0$, is shown by a broken line (cf. Sect. 4.1), and that for a dynamo without inflow, i.e. $R_{u}=0$, is shown by a dotted line (cf. Sect. 4.2). The field approaches that found in the absence of dynamo action as $\mathcal{B}$ increases, and that of a pure dynamo (without inflow) as $\mathcal{B}$ decreases. Some solutions shown in panel c) are oscillatory, and then the solid and dotted lines show the range of the field variation over a period.

\subsubsection{Temporal variation}

Since the boundary conditions at $r=R$ are inhomogeneous when $\mathcal{B} \neq 0$, Eqs. (5) and (6) do not admit exponentially growing solutions even at the linear stage when $B^{2} \ll 4 \pi k \rho v_{\mathrm{t}}^{2}$. However, the quadrupolar part of the magnetic field, maintained by the dynamo, exhibits initial exponential growth at an early stage followed by saturation, as might be expected for normal dynamo action. The dipolar dynamo modes decay at the values of $R_{\alpha}$ and $R_{\omega}$ considered here, so the dipole magnetic field within the
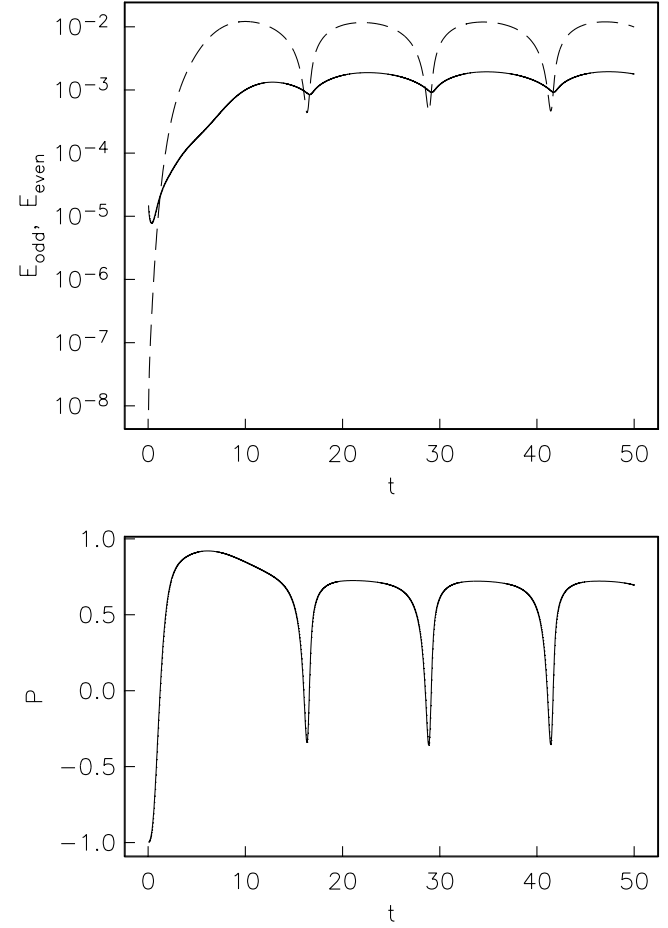

Fig. 8. Upper panel: Magnetic energies in the odd (solid) and even (broken) parts of magnetic field in the whole computational domain for $\eta_{0}=10^{26} \mathrm{~cm}^{2} \mathrm{~s}^{-1}, \mathcal{B}=10^{-3}$ and $R_{u}=6$ (see also Table 1). The solution is oscillatory but its initial stage of growth is similar to that of non-oscillatory solutions. Lower panel: Global parity of magnetic field versus time. The even part of the magnetic field oscillates at a much larger amplitude than the odd part. Time is measured in units of $7.5 \times 10^{8} \mathrm{yr}$.

disc only grows due to advection (perhaps modified by semi-dynamo action), and this growth is non-exponential. This is illustrated in Fig. 8 where we show the evolution with time of the energies in the even and odd parts of the magnetic field for $R_{u}=6, \mathcal{B}=10^{-3}$. The curve for the even field deviates from a straight line in this log-linear plot because of the contribution of the halo to the total energy (the exponential growth is confined to the disc). The solution is oscillatory, and the quadrupolar part has a much larger amplitude of oscillations than the dipolar; this is natural as the oscillations are definitely driven by the dynamo. The initial growth before the onset of the oscillations is fairly similar to that found in non-oscillatory solutions.

The time for the steady state configurations to become established will depend on the initial conditions. We have chosen what are arguably the least favourable, with no flux initially penetrating the disc. The time to reach a steady state is then of the order of $10^{10} \mathrm{yr}$. It is plausible that galaxies trap some poloidal flux at all radii as they form. Thus the timescale for our models to reach a steady state should be regarded as an upper limit. Moreover, changes in the disc, being directly driven by the inflow, will occur more rapidly if the inflow speed exceeds about $1 \mathrm{~km} \mathrm{~s}^{-1}$, whereas the halo adjusts on a slower, diffusive, timescale; 
Table 2. Summary of results for calculations with $\eta_{0}=3 \times 10^{26} \mathrm{~cm}^{2} \mathrm{~s}^{-1}$, the central gas surface density $\sigma(0)=5 M_{\odot} \mathrm{pc}-2$, and $R_{u}=0,2,5$, and 10 . The format is similar to that of Table 1 . When $R_{u}=0, \mathcal{B}=0$, we obtain $\left(P, P_{i}, E\right)=(+1,+1,0.90)$.

\begin{tabular}{|c|c|c|c|c|c|c|}
\hline $\mathcal{B}$ & 1 & $10^{-1}$ & $10^{-2}$ & $10^{-3}$ & $10^{-4}$ & 0 \\
\hline \multicolumn{7}{|c|}{$R_{u}=2$} \\
\hline$P$ & -1 & -0.7 & 0.57 & 0.99 & +1 & +1 \\
\hline$P_{\mathrm{i}}$ & -1 & -0.995 & -0.63 & 0.91 & +1 & +1 \\
\hline$E$ & $2.6 \times 10^{2}$ & 1.92 & 0.78 & 0.62 & 0.60 & 0.60 \\
\hline$E_{\text {adv }}$ & $2.8 \times 10^{2}$ & 2.8 & $2.8 \times 10^{-2}$ & $2.8 \times 10^{-4}$ & $2.8 \times 10^{-6}$ & 0 \\
\hline$\varphi$ & 0.0080 & 0.0057 & 0.020 & 0.024 & 0.023 & - \\
\hline $\bar{B}_{z, \mathrm{c}}$ & 20.0 & 1.43 & 0.50 & 0.060 & 0.0058 & 0 \\
\hline \multicolumn{7}{|c|}{$R_{u}=5$} \\
\hline$P$ & -1 & -1 & -0.18 & 0.97 & +1 & +1 \\
\hline$P_{\mathrm{i}}$ & -1 & -1 & -0.82 & 0.80 & +1 & +1 \\
\hline$E$ & $2.8 \times 10^{3}$ & 2.0 & 0.62 & 0.26 & 0.26 & 0.26 \\
\hline$E_{\mathrm{adv}}$ & $3.0 \times 10^{3}$ & $3.0 \times 10^{1}$ & $3.0 \times 10^{-1}$ & $3.0 \times 10^{-3}$ & $3.0 \times 10^{-5}$ & 0 \\
\hline$\varphi$ & 0.064 & 0.018 & 0.077 & 0.085 & 0.084 & - \\
\hline $\bar{B}_{z, \mathrm{c}}$ & 160. & 4.50 & 1.93 & 0.21 & 0.021 & 0 \\
\hline \multicolumn{7}{|c|}{$R_{u}=10$} \\
\hline$P$ & -1 & -1 & -1 & -1 & -1 & - \\
\hline$P_{\mathrm{i}}$ & -1 & -1 & -1 & -1 & -1 & - \\
\hline$E$ & $7.0 \times 10^{3}$ & $3.2 \times 10^{1}$ & $3.0 \times 10^{-2}$ & $2.6 \times 10^{-4}$ & $2.6 \times 10^{-6}$ & decay \\
\hline$E_{\mathrm{adv}}$ & $7.2 \times 10^{3}$ & $7.2 \times 10^{1}$ & $7.2 \times 10^{-2}$ & $7.2 \times 10^{-4}$ & $7.2 \times 10^{-6}$ & 0 \\
\hline$\varphi$ & 0.19 & 0.13 & 0.032 & 0.022 & 0.022 & - \\
\hline $\bar{B}_{z, \mathrm{c}}$ & 475. & 32.5 & 0.80 & 0.055 & 0.0018 & 0 \\
\hline
\end{tabular}

therefore, a stationary state can be established in the disc over a shorter time scale.

\subsection{Enhanced magnetic diffusivity: $\eta_{0}=3 \times 10^{26} \mathrm{~cm}^{2} \mathrm{~s}^{-1}$}

We then studied a model with an increased magnetic diffusivity in the disc, $\eta_{0}=3 \times 10^{26} \mathrm{~cm}^{2} \mathrm{~s}^{-1}$ and thus also in the halo. As in the previous section, we adopted $\sigma=5 M_{\odot} \mathrm{pc}^{-2}$ in $r<2 \mathrm{kpc}$. Now field amplification by differential rotation is weaker because of enhanced diffusion, $R_{\omega}=6.25$, and so the critical value of $R_{\alpha}$ is significantly increased; we took $R_{\alpha}=2$, again a somewhat supercritical value for dynamo action. With these parameter values, $R_{u}=1$ corresponds to an inflow speed of about $1 \mathrm{~km} \mathrm{~s}^{-1}$ near the Sun. Our results are presented in Table 2. Comparing these with those in Table 1, we see a broadly comparable pattern in the results when $R_{u} \leq 5$, with advection significantly affecting the dynamo field when $\mathcal{B} \gtrsim 10^{-3}$. Arguably, in this case advection is somewhat less effective, consistent with what can be expected for the larger magnetic diffusivity. In particular, the concentration of the vertical magnetic flux towards the disc axis, $\varphi$, increases as $\mathcal{B}$ decreases for $10^{-4}<\mathcal{B}<10^{-1}$, and decreases for smaller values of $\mathcal{B}$.
For $R_{u}=10$, the situation is rather different. Now the dynamo is not excited when $\mathcal{B}=0$. Even so, dynamo action plays a role; in the range $10^{-4} \leq B_{\text {ext }} \leq 1$, we find that the saturated magnetic energy is less than that of the advected field, i.e. $E<E_{\mathrm{adv}}$, by a factor of approximately 3 for the smaller values of $\mathcal{B}$. The field always has a dipolar parity, $P=-1$, not typical of the dynamo configurations with moderate dynamo numbers explored here.

When a quadrupolar field is not excited, the dipolar field energy in the presence of an imposed flux and dynamo action is reduced from that when the dynamo is turned off. This leads us to deduce that a form of semi-dynamo, exciting an odd parity field, is operating (cf. Drobyshevski 1977); i.e. subcritical dynamo action that is dependent on an external source of flux. The fields so generated, although of the same, odd, parity as the advected field, nevertheless interact destructively with the advected field, reducing the total energy below that of the purely advected field (i.e. that found with $R_{\alpha}=0$ ).

\section{A Milky Way model with high central density}

Here we examine a model more specifically relevant to the Milky Way, with $\sigma(0)=300 M_{\odot} \mathrm{pc}^{-2}$ and $\eta_{0}=$ $10^{26} \mathrm{~cm}^{2} \mathrm{~s}^{-1}$. We take $R_{u}=3$, so the inflow velocity near 


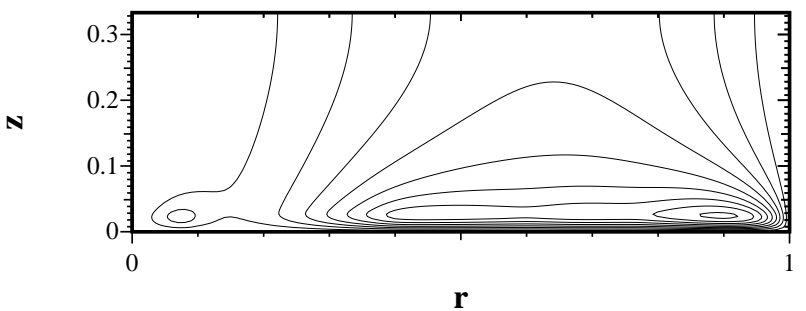

(a)

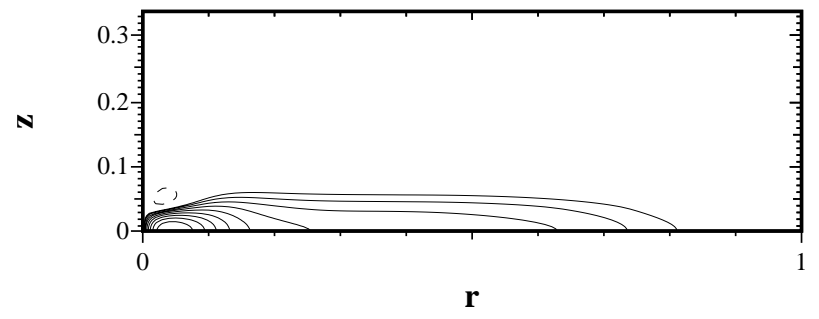

(b)

Fig. 9. a) The poloidal field lines equally spaced in poloidal flux and b) contours of toroidal field with contour levels starting at unity and increasing by a factor of 1.5 in toroidal field strength between contours, for the pure dynamo calculation of Sect. 6.1 with $R_{u}=0, R_{\omega}=18.75, \eta_{0}=10^{26} \mathrm{~cm}^{2} \mathrm{~s}^{-1}$, $R_{\alpha}=0.5$ and $\sigma(0)=300 M_{\odot} \mathrm{pc}^{-2}$. The field has an even (quadrupolar, $P=+1$ ) structure with respect to the midplane $(z=0)$, so only the upper half space is shown.

the Sun is about $1 \mathrm{kms}^{-1}$. The reference model with no dynamo action is as described in Sect. 4.1 - in this linear calculation the gas density has no role. We describe in Sect. 6.1 a nonlinear dynamo model with no radial flow, before giving our results for models with inflow and a range of $\mathcal{B}$ values.

\subsection{The basic dynamo model}

In Fig. 9 we show the poloidal field lines and toroidal field contours for the basic dynamo model $\left(R_{u}=0, \mathcal{B}=0\right)$. Comparison with Fig. 3 shows that the toroidal field is much more strongly concentrated to the galactic centre than in the models of Sect. 5 even though its magnitude at $r \simeq R_{\odot}$ remains approximately the same. This is a natural consequence of the strongly enhanced central density, and correspondingly increased field strength $B_{0}$ at which the dynamo saturates.

\subsection{Dynamos with imposed flux through the midplane}

We first discuss results with $R_{\alpha}=0.5$, the value used in Sect. 5.1. In Fig. 10 we present the field structure (poloidal field lines and contours of toroidal field) for a model with $\mathcal{B}=10^{-3}$. Marked departures from pure symmetry are visible; here $P=0.87$ and $P_{i}=0.82$, although these are not as strong as for the corresponding model with small central density, see Fig. 4. The toroidal field is now much more strongly concentrated to the galactic centre

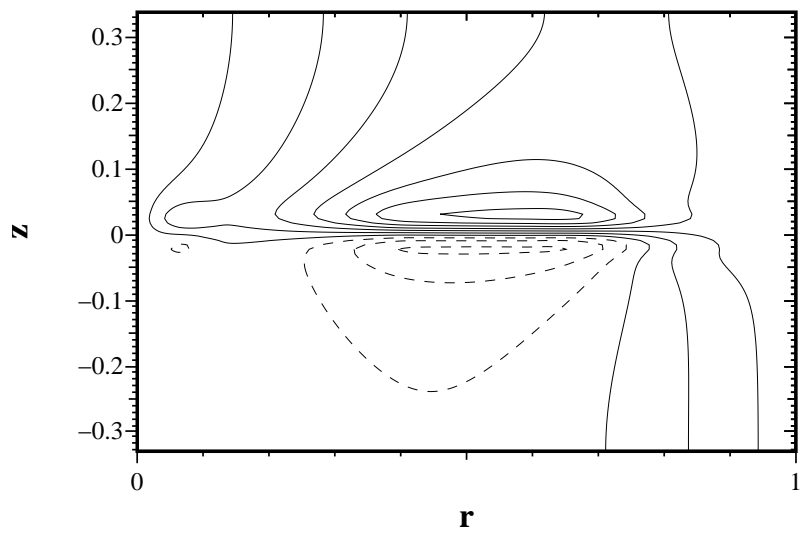

(a)

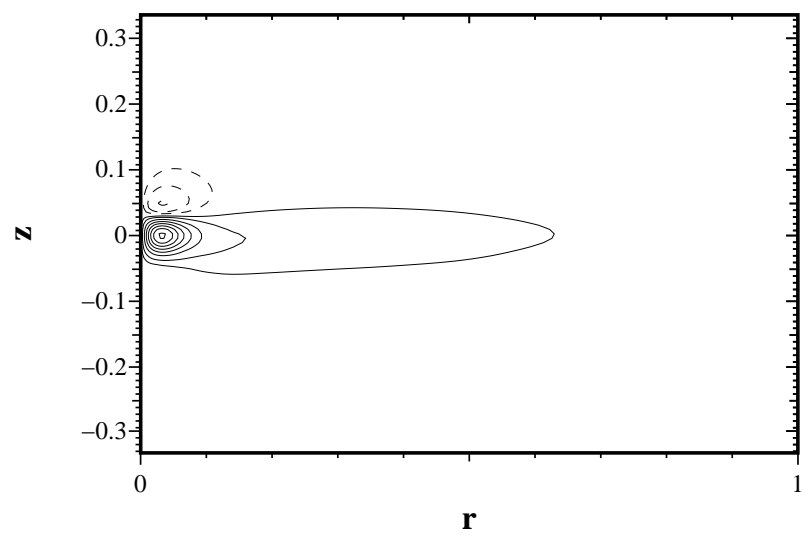

(b)

Fig. 10. a) The poloidal field lines equally spaced in poloidal flux and b) contours of toroidal field with contour levels starting at unity and increasing by a factor of 2.6 in toroidal field strength between contours, for the calculation of Sect. 6.2 with $R_{\alpha}=0.5, R_{u}=3, R_{\omega}=18.75, \eta_{0}=10^{26} \mathrm{~cm}^{2} \mathrm{~s}^{-1}, R_{\alpha}=0.5$, $\sigma(0)=300 M_{\odot} \mathrm{pc}^{-2}$ and $\mathcal{B}=10^{-3}$. Continuous and broken contours refer to positive and negative values respectively.

with $\left.B\right|_{r=0.02} /\left.B\right|_{r=R_{\odot}} \simeq 20$. Our results for $0 \leq \mathcal{B} \leq 1$ are given in Table 3 . We see a broad similarity with the results of Sects. 5.1 and 5.2. The steady-state magnetic energy decreases with increasing inflow rate, cf. Eq. (9). As $\mathcal{B}$ increases at fixed $R_{u}$, then $E_{\text {dyn }} \leq E \leq E_{\text {adv }}$ and both $P$ and $P_{i}$ decrease monotonically, so the field becomes more dipole-like both globally and in the inner $300 \mathrm{pc}$. The only significant difference to the results found above with the smaller value of the central density is that $P_{i}<P$ for intermediate values of $\mathcal{B}$, i.e. the central field is less dipolelike than the global field. Further (but related) we note that for these intermediate values of $\mathcal{B}, \varphi$ is smaller than for the corresponding model with $\sigma(0)=5 M_{\odot} \mathrm{pc}^{-2}$ (see Table 1). We suspect this is because the stronger dynamo generated field now present in the central regions is better able to interact destructively with the advected field.

We also experimented with a reduction of the truncation radius $r_{u}$ for the velocity profile to 0.05 (corresponding to $0.75 \mathrm{kpc}$ ) from our standard value $r_{u}=0.10$ (see 
Table 3. Summary of results for $\eta_{0}=10^{26} \mathrm{~cm}^{2} \mathrm{~s}^{-1}, R_{u}=3$, and the central gas surface density $\sigma(0)=300 M_{\odot} \mathrm{pc} c^{-2}$. The format is similar to that of Table 1 . For $R_{u}=0, \mathcal{B}=0$ we obtain $\left(P, P_{i}, E\right)=(+1,+1,0.84)$ when $R_{\alpha}=0.5$. and $(+1,+1,0.17)$ for $R_{\alpha}=0.25$.

\begin{tabular}{|c|c|c|c|c|c|c|c|c|c|}
\hline $\mathcal{B}$ & 1 & $10^{-1}$ & $6 \times 10^{-2}$ & $3 \times 10^{-2}$ & $2 \times 10^{-2}$ & $10^{-2}$ & $10^{-3}$ & $10^{-4}$ & 0 \\
\hline \multicolumn{10}{|c|}{$R_{\alpha}=0.5$} \\
\hline$P$ & -1 & -1 & -1 & -0.86 & -0.69 & -0.35 & 0.87 & 0.990 & +1 \\
\hline$P_{\mathrm{i}}$ & -1 & -1 & -1 & -0.61 & -0.12 & 0.07 & 0.82 & 0.998 & +1 \\
\hline$E$ & $7.66 \times 10^{3}$ & 48.0 & 4.6 & 0.80 & 0.88 & 0.80 & 0.44 & 0.42 & 0.42 \\
\hline$E_{\mathrm{adv}}$ & $7.68 \times 10^{3}$ & $7.68 \times 10^{1}$ & 27.6 & 6.91 & 3.07 & 0.768 & $7.68 \times 10^{-3}$ & $7.68 \times 10^{-5}$ & 0 \\
\hline$\varphi$ & 0.026 & 0.021 & 0.0089 & 0.0032 & 0.0051 & 0.0080 & 0.054 & 0.067 & - \\
\hline $\bar{B}_{z, \mathrm{c}}$ & 65.0 & 5.2 & 1.3 & 0.24 & 0.26 & 0.20 & 0.14 & 0.017 & 0 \\
\hline \multicolumn{10}{|c|}{$R_{\alpha}=0.25$} \\
\hline$P$ & -1 & & & -1.0 & -0.996 & -0.56 & 0.91 & 0.999 & +1 \\
\hline$P_{\mathrm{i}}$ & -1 & & & -1.0 & -0.992 & 0.12 & 0.94 & 0.999 & +1 \\
\hline$E$ & $7.64 \times 10^{3}$ & & & 1.03 & 0.38 & 0.18 & 0.11 & 0.11 & 0.11 \\
\hline$E_{\mathrm{adv}}$ & $7.68 \times 10^{3}$ & & & 6.91 & 3.07 & 0.768 & $7.68 \times 10^{-3}$ & $7.68 \times 10^{-5}$ & 0 \\
\hline$\varphi$ & 0.026 & & & 0.0065 & 0.0030 & 0.0069 & 0.025 & 0.027 & - \\
\hline $\bar{B}_{z, \mathrm{c}}$ & 65.0 & & & 0.49 & 0.15 & 0.17 & 0.063 & 0.0067 & 0 \\
\hline
\end{tabular}

Sect. 3.2). This resulted in an increase of $\varphi$ by a factor of about 2 when $\mathcal{B}=10^{-3}$, and of about 2.5 when $\mathcal{B}=0.1$. We consider this to give an estimate of the uncertainties in our model.

Given the importance of the destructive interaction of dynamo and advected fields, we also investigated a model with weaker dynamo action, taking $R_{\alpha}=0.25$. These results are also presented in Table 3 . The change of the diagnostic parameters as $\mathcal{B}$ increases follows a similar pattern as described above when $R_{\alpha}=0.5$, except that the transition to a dipole-like field structure occurs at smaller values of $\mathcal{B}$. In particular, we note that except when $\mathcal{B}=3 \times 10^{-2}$ (when already $P \approx-1$ ), the mean central field $\bar{B}_{z, c}$ is smaller than when $R_{\alpha}=0.5$. We conclude that the dipolar magnetic field at the disc centre is supported by dynamo action even though the latter favours quadrupolar magnetic configurations. This may be considered as another manifestation of semi-dynamo action, cf. Sect. 5.2.

\section{Discussion and conclusions}

\subsection{Implications for magnetic field in the Galactic centre}

An unexpected result of our simulations is that the relative concentration of vertical flux in the central region, $\varphi$, does not increase monotonically with the vertical flux $\mathcal{B}$. This effect is especially strong for moderate inflow speeds. For example, in Table 3 with $R_{u}=3, R_{\alpha}=0.5$, the fraction of the vertical flux stored in the inner 300 pc grows by a factor of 20 as the magnitude of the flux parameter $\mathcal{B}$ decreases from $3 \times 10^{-2}$ to $10^{-4}$. Increasing the central density enhances the central quadrupolar field, but reduces the dipolar vertical field there.
Our model, with a high turbulent magnetic diffusion in the halo, favours advection (also the field is not anchored high above the disc). Nevertheless, our results indicate that the nonlinear interaction with the dynamo limits the vertical magnetic field at the centre to relatively low values even when both the advection and the captured vertical field are rather strong. In Tables 1, 2 and 3 we give the values of $\bar{B}_{z, \mathrm{c}}=\mathcal{B} \varphi / r_{\mathrm{i}}^{2}=2500 \mathcal{B} \varphi$, the dimensionless mean vertical field within the inner $300 \mathrm{pc}$ radius (i.e. $r \leq r_{\mathrm{i}}$ ), the overbar denoting a horizontal average. For the high central density model of Sect. 6 (Table 3 ), the mean central field $\bar{B}_{z, \mathrm{c}}$ is approximately constant in an interval $10^{-3} \lesssim \mathcal{B} \lesssim 3 \times 10^{-2}$. Quite generally, $\bar{B}_{z, \mathrm{c}} \lesssim(10-20) k^{1 / 2} \mu \mathrm{G}$ for $\mathcal{B}<0.1$ and realistic values of $R_{u}$, and generally decreases with $\mathcal{B}$. (Note that this is a higher estimate than might be deduced from Fig. 11, where $\mathcal{B}=10^{-3}$.)

The estimated magnitude of $\bar{B}_{z, \mathrm{c}}$ may be further increased by a factor of 2-3 because of uncertainty in the inflow speed in the central region. However, any increased turbulent velocity in $r<300 \mathrm{pc}$ (not allowed for in our models) would result in larger values of $B_{0}$, thus both enhancing the quadrupole magnetic field due to the dynamo and increasing the turbulent diffusivity. The latter would hamper advection of the vertical magnetic field in the central regions, and could possibly compensate any increase in $\bar{B}_{z, \mathrm{c}}$. Another possibility is that $\alpha_{0}$ may be underestimated at small radii in our model if $\alpha_{0} \propto \Omega$; this would make the dynamo stronger in the central regions, again favouring the generation of quadrupolar magnetic fields there. The resulting average vertical field at $r<300 \mathrm{pc}$ would then remain not stronger than $0.01 \mathrm{mG}$, two orders of magnitude weaker than required if the magnetic field illuminated in the filaments fills the volume. 


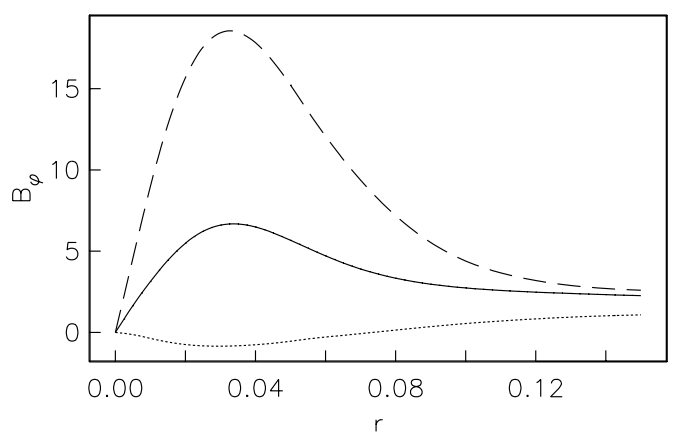

(a)

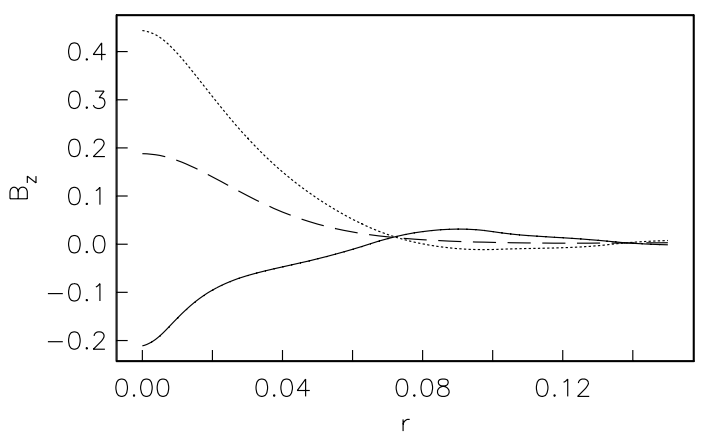

(b)

Fig. 11. Radial profiles of the azimuthal a) and vertical $\mathbf{b}$ ) magnetic field components at $z=-500 \mathrm{pc}$ (solid), $z=0$ (dashed) and $z=500 \mathrm{pc}$ (dotted) in the inner $2 \mathrm{kpc}$ in a model with $R_{u}=3, R_{\alpha}=0.5, \eta=10^{26} \mathrm{~cm}^{2} \mathrm{~s}^{-1}$, the central gas surface density $300 M_{\odot} \mathrm{pc}^{-2}$ and $\mathcal{B}=10^{-3}$ in dimensionless units. Magnetic field is in units of $8 k^{1 / 2} \mu \mathrm{G}$, and radius in units of $15 \mathrm{kpc}$.

We show in Fig. 11 the radial variation of the azimuthal and vertical magnetic field components in a model with a radial velocity of about $1 \mathrm{~km} \mathrm{~s}^{-1}$ near the Sun, where the degree of concentration of $B_{z}$ to the centre, $\varphi$, is nearly the strongest among the models studied. The profiles are shown for $z=0$ and \pm 500 pc to provide further indication (in addition to the parity parameter $P_{\mathrm{i}}$ introduced above) of the degree of symmetry of the vertical profiles with respect to the midplane. Even though the dipolar component [with $B_{\phi}(z)=-B_{\phi}(-z)$ and $B_{z}(z)=B_{z}(-z)$ ] is significant (especially in the azimuthal field), the dynamo-favoured quadrupolar component [with $B_{\phi}(z)=B_{\phi}(-z)$ and $B_{z}(z)=-B_{z}(-z)$ ] is stronger. Correspondingly, the parity in the inner $300 \mathrm{pc}$ is rather close to unity (see Table 3). Further details for these high central density models are presented in Table 3 and in Fig. 12.

The vertical magnetic field shown in Fig. 11 has a maximum at $r=0$; this is a typical feature. However, the maximum value is $B_{z} \simeq 2 \times 10^{-6} \mathrm{G}$ at $r, z=0$ (taking $k=1$ ), which is significantly below the level corresponding to equipartition with kinetic energy, $B_{0} \simeq 10^{-5}-10^{-4} \mathrm{G}$. The azimuthal field vanishes at $r=0$ (because of the azimuthal symmetry) and reaches a maximum at a distance

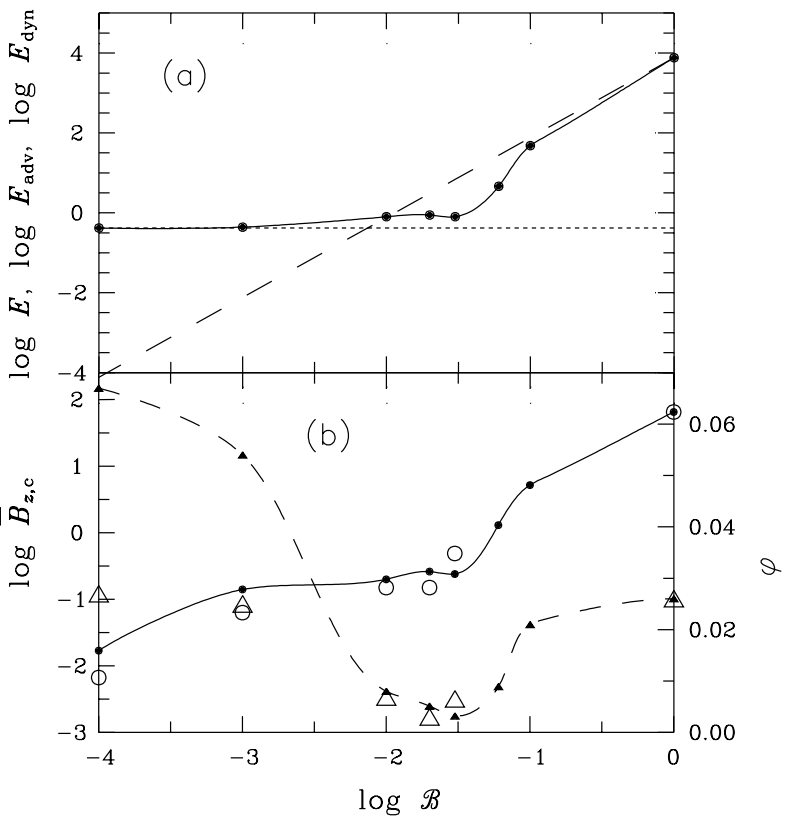

Fig. 12. a) The total magnetic energy $E$ versus the captured flux parameter $\mathcal{B}$ in the model of Sect. 6 is shown by a solid line for $R_{\alpha}=0.5$. The advected field energy and dynamo energy are shown dashed and dotted as in Fig. 7. b) The mean vertical magnetic field (solid) and the relative flux $\varphi$ through the inner $300 \mathrm{pc}$ radius (broken) versus $\mathcal{B}$ for $R_{\alpha}=0.5$. The corresponding results for weaker dynamo action, with $R_{\alpha}=0.25$, are shown with open circles and triangles, respectively. Magnetic field is measured in units of $8 k^{1 / 2} \mu \mathrm{G}$.

of about $500 \mathrm{pc}$ from the centre; the maximum value of about $1.5 \times 10^{-4} \mathrm{G}$ (again taking $k=1$ ) is slightly above the equipartition level.

To limit the range of $\mathcal{B}$ of physical interest, we can apply three constraints to our models. On observational grounds we can probably rule out solutions with $E \gg$ $E_{\text {dyn }}$, simply because $E_{\text {dyn }}$ is close to the kinetic energy of the turbulence and corresponds well to the field strengths observed in the outer parts of the Milky Way and other galaxies. Thus larger values of the energy would imply strongly super-equipartition large scale (i.e. regular) fields throughout the galactic disc, for which there is no evidence in normal spiral galaxies (e.g., Beck 2000). This constraint thus suggests that we should probably restrict our attention to values $\mathcal{B} \lesssim 5 \times 10^{-2}$.

Secondly, there is evidence to suggest that, in common with external spiral galaxies, the global magnetic field of the Milky Way should be of even (quadrupolar) parity (Frick et al. 2000 and references therein). If conservatively we require $P>0$, then we need $\mathcal{B} \lesssim 10^{-2}$.

Thirdly, if we estimate the maximum large-scale magnetic field strength in the protogalactic gas to be $\lesssim 3 \times$ $10^{-12} \mathrm{G}$ (Sciama 1994), then in contracting from $100 \mathrm{kpc}$, an estimated radius for the protogalaxy, to the presentday cylindrical radius of $20 \mathrm{kpc}$ without loss of flux, we obtain a mean vertical field through the disc of less than about $10^{-10} \mathrm{G}$, i.e. $\mathcal{B} \lesssim 10^{-5}$. This is a negligibly weak field in the present context. For a galaxy formed in a rich 
cluster of galaxies (though mostly populated by ellipticals rather than spirals), the external field could be a fraction of $10^{-6} \mathrm{G}$, corresponding to $\mathcal{B} \simeq 0.1-0.01$ in dimensionless units. This is clearly a very generous upper limit for the Milky Way.

These considerations all suggest that we need only consider models from Table 3 with $\mathcal{B} \lesssim 0.05$ (of order $10^{-7} \mathrm{G}$ ), and possibly only those with $\mathcal{B} \lesssim 0.01$, or even much less. This implies that $\bar{B}_{z, \mathrm{c}} \lesssim 1.0$, that is the mean vertical field near the galactic centre is less than about $10 k^{1 / 2} \mu \mathrm{G}$. Uncertainties associated with the choice of the inflow velocity cut off parameter $r_{u}$ (see Sect. 6.2) seem unlikely to change this estimate by more than a factor of 2 or 3 . We also note that the same arguments applied to the low central density models of Table 1 with $R_{u}=3$ lead to similar estimates.

Dynamo action is not the only obstacle to the accumulation of dipolar magnetic flux in the Galactic centre. Even the pure field advection models with the lower value $\eta_{0}=10^{26} \mathrm{~cm}^{2} \mathrm{~s}^{-1}$ and $R_{\alpha}=0$ as in Sect. 4.1 (ignoring for a moment that these models have strictly odd parity, $P=-1$ and so do not reproduce magnetic field structure near the Sun), and $\mathcal{B} \lesssim 10^{-2}$ again yield $\bar{B}_{z, c} \lesssim 1$, and so the mean central field is $\lesssim 10 \mu \mathrm{G}$.

The models explored here indicate that a vertical magnetic field of an average milliGauss strength cannot be accumulated in the centre of the Galactic disc. The striking dipolar symmetry implied by the straight shape of the magnetic filaments in the Galactic centre also does not develop in our models. Our model is not designed to produce such fine filamentary structure. However, unless the filling factor of the magnetic field is extremely small (i.e. the filaments are not parts of a more uniform field illuminated only intermittently - see Morris \& Serabyn 1996 for a discussion), there is certainly insufficient vertical flux present in our models.

We emphasize that we have only considered magnetic fields resulting from Galactic-scale accretion and dynamo action. It cannot be excluded that the central regions of the Galaxy represent an autonomous system relatively weakly connected with the outer parts, at least in terms of magnetic field. In that case, it would be necessary to develop a separate, specific MHD model (allowing for dynamo action) for this region. What we have shown, however, is that the observed milligauss vertical magnetic fields within the inner 300 pc would be difficult to explain by the accumulation of magnetic flux from the outer parts if dynamo is active in the Galaxy, even if the total vertical magnetic flux is conserved. This conclusion seems rather general and independent of the actual dynamo mechanism.

Our conclusions are in variance with those of Chandran et al. (2000), principally because these authors assume the large-scale magnetic field to be frozen into the interstellar gas. They argue that the dissipation of magnetic energy in the hot gas, expected to occur at the proton gyroradius scale, can take as long as $10^{14} \mathrm{yr}$. However, the large-scale magnetic field is more plausibly anchored in the warm phase (Beck et al. 1996) which has larger filling factor and can be pervasive, whereas the hot gas is buoyant and can leave the disc in a short time to be confined to isolated regions in the disc. Furthermore, the large-scale magnetic field is affected mostly by turbulent magnetic diffusion which is determined by the energy-range scale of turbulence about $100 \mathrm{pc}$, and the velocity at this scale, rather than by the dissipation scale of the turbulence. Moreover, mean-field dynamo action does not require genuine turbulence at all (i.e. any details of turbulent energy cascade are unimportant) - what is needed is merely random, mirrorasymmetric, flow. Just as fundamentally, our results indicate that dynamo action in itself acts to inhibit advection of field: this is the reverse side of inflow inhibiting dynamo action in the absence of any external magnetic field. There is no reason to believe that this result is dependent on the detailed nature of the dynamo (e.g. the $\alpha$-effect).

Chandran et al. (2000) similarly argue that gravity and tension of the large-scale magnetic field will suppress the random nature of interstellar gas motions thereby suppressing turbulent diffusion. It is, however, known that turbulent diffusion remains effective even in stronger gravity fields, e.g., in the solar convective zone. Magnetic fields undoubtedly suppress turbulent diffusion together with the $\alpha$-effect (e.g., Krause \& Rädler 1980), but these effects plausibly occur simultaneously, so that dynamo action, controlled by the dynamo number $D \propto \alpha / \eta^{2}$, can continue (e.g. Moss in Mestel 1999, p. 222; Brandenburg 2000.) Moreover, a large-scale magnetic field can even enhance turbulent magnetic diffusivity in some models (Brandenburg \& Subramanian 2000). Efficient magnetic turbulent diffusion does not imply that gas will be removed from the disc together with magnetic fields (Chandran et al. 2000) as gas freely slips down the vertical sections of tangled magnetic lines.

Altogether, we conclude that there are no compelling reasons to consider the large-scale galactic magnetic field to be frozen into the interstellar gas and the dynamo action to be suppressed. We reiterate that intense turbulent motions are observed in the interstellar medium, and these motions cannot avoid tangling the large-scale magnetic field.

\subsection{More general implications for galactic dynamo theory}

Our system also demonstrates several features of general interest in dynamo theory. In the main they have been described above, and are merely recapitulated here.

For large enough inflow velocities, the dynamo is killed in the absence of an imposed vertical flux, supporting the conclusions of Moss et al. (2000). As this situation is approached with increasing $R_{u}$, the dynamo may become oscillatory at relatively low energy; these oscillations appear only in the fully $2 \mathrm{D}$ model studied here, and not in the approximate dynamo solutions discussed by Moss et al. (2000). For small and moderate $R_{u}$, the steady-state magnetic field scales as $\left(1-c R_{u}\right)^{1 / 2}$ with $c$ close to $1 / 6$. 
In the presence of a vertical flux $(\mathcal{B} \neq 0)$, semi-dynamo action may occur; this is explicit when $R_{u}$ is large enough that pure dynamo action is suppressed, and the steadystate magnetic energy still differs significantly from that obtained with pure advection, i.e. without any $\alpha$-effect.

In general the energy of the hybrid field (from dynamo action and advection) is not the sum of the energies of the corresponding pure dynamo and purely advected fields - for larger $\mathcal{B}$ values, $E<E_{\text {adv }}$ even in cases where a dynamo is not excited when $\mathcal{B}=0$. This is somewhat reminiscent of a result found by Moss (1996) when investigating stellar dynamos with an imposed background field. We find that, as long as $E_{\text {adv }} \gtrsim E_{\text {dyn }}$, the (true) dynamo generated fields interact destructively with the advected field, i.e. resulting in $E<E_{\text {adv }}$. In contrast, the interaction is constructive when $E_{\text {adv }}<E_{\text {dyn }}$, i.e. $E>E_{\text {adv }}+E_{\text {dyn }}$ in the stationary state.

Although it seems plausible that a large enough vertical flux and inflow velocity could seriously inhibit dynamo action via $\alpha$-quenching caused by the advected vertical field, we do not see unambiguous evidence for this in our models. For large enough $R_{u}$, the dynamo is in any case suppressed by essentially linear effects (even when $\mathcal{B}=0$ ). However, as $\mathcal{B}$ increases, there is evidence $\left(E<E_{\text {adv }}\right)$ that the advected field promotes some form of dynamo action, even in the extreme case $R_{u}=10, \mathcal{B}=1$ (Table 2). It is clear that the effects of nonlinearity in this dynamo system can take subtle forms.

We have here discussed only dynamo systems with $R_{\alpha}>0$, in which the basic field generated is of even (quadrupolar) parity, as believed to be appropriate to spiral galaxies. If in accretion discs $R_{\alpha}$ is negative (Brandenburg et al. 1995; Rüdiger \& Pipin 2000), then a dipolar (odd) parity dynamo field is generated. The outcome of the interaction of such a dynamo with a trapped vertical flux of the same odd parity is unclear. We intend to address this question elsewhere.

Acknowledgements. We acknowledge support from PPARC Grant PPA/G/S/1997/00284 and NATO Linkage Grant PST.CLG 974737.

\section{References}

Bardou, A., \& Heyvaerts, J. 1996, A\&A, 307, 1009 Beck, R. 2000, Phil. Trans. R. Soc. Lond., 358, 777 Beck, R., Brandenburg, A., Moss, D., Shukurov, A., \& Sokoloff, D. D. 1996, ARA\&A, 34, 155

Blackman, E. G., \& Field, G. B. 2000, ApJ, 534, 984

Blandford, R. D., \& Payne, D. R. 1982, MNRAS, 199, 883

Boyer, D. W., \& Levy, E. H. 1984, ApJ, 277, 848

Brandenburg, A. 2000, ApJ, in press [astro-ph/0006186]

Brandenburg, A., Nordlund, Å., \& Stein, R. F. 1995, ApJ, 446, 741

Brandenburg, A., \& Subramanian, K. 2000, A\&A, 361, L33

Brandenburg, A., Donner, K.-J., Moss, D., et al. 1992, A\&A, 259,453

Brandenburg, A., Donner, K.-J., Moss, D., et al. 1993, A\&A, 271,36
Burton, W. B., \& Gordon, M. A. 1978, A\&A, 202, 30

Chandran, B. D. G., Cowley, S. C., \& Morris, M. 2000, ApJ, 528,723

Dame, T. M. 1992, in Back to the Galaxy, ed. S. Holt, \& F. Verter, AIP Conf. Ser., 278 (AIP, New York), 267

Drobyshevski, E. M. 1977, ApSS, 46, 41

Frick, P., Stepanov, R., Shukurov, A., \& Sokoloff, D. 2000, MNRAS, in press [astro-ph/0012459]

Gabov, A. S., Sokoloff, D. D., \& Shukurov, A. 1996, Astron. Rep., 40, 463

Howard, A. M., \& Kulsrud, R. M. 1997, ApJ, 483, 648

Kleeorin, N., Moss, D., Rogachevskii, I., \& Sokoloff, D. 2000, A\&A, 361, L5

Krause, F., \& Rädler, K.-H. 1980, Mean-Field Magnetohydrodynamics and Dynamo Theory (Pergamon Press, Oxford)

Kronberg, P. P. 1994, Rep. Prog. Phys., 57, 325

Kulsrud, R. M. 1999, ARA\&A, 37, 37

Lacey, C. G., \& Fall, S. M. 1985, ApJ, 290, 154

Lubow, S. H., Balbus, S. A., \& Cowie, L. L. 1986, ApJ, 309, 496

Lubow, S. H., Papaloizou, J. C. B., \& Pringle, J. E. 1994, MNRAS, 267, 235

Mestel, L. 1999, Stellar Magnetism (Clarendon Press, Oxford)

Minter, A. H., \& Spangler, S. R. 1996, ApJ, 458, 194

Moffatt, H. K. 1978, Magnetic Field Generation in Electrically Conducting Fluids (Cambridge Univ. Press, Cambridge)

Morris, M., \& Serabyn, E. 1996, ARA\&A, 34, 645

Moss, D. 1996, A\&A, 305, 140

Moss, D., Shukurov, A., \& Sokoloff, D. 1999, A\&A, 343, 120

Moss, D., Shukurov, A., \& Sokoloff, D. 2000, A\&A, 358, 1142

Ouyed, R., \& Pudritz, R. E. 1997, ApJ, 482, 712

Parker, E. N. 1979, Cosmic Magnetic Fields (Clarendon Press, Oxford)

Parker, E. N. 1992, ApJ, 401, 137

Pitts, E., \& Tayler, R. J. 1996, MNRAS, 280, 1101

Poezd, A., Shukurov, A., \& Sokoloff, D. 1993, MNRAS, 264, 285

Pudovkin, M. I., \& Benevolenskaya, E. E. 1984, Sov. Astron., 28,458

Reyes-Ruiz, M., \& Stepinski, T. F. 1996, ApJ, 459, 653

Reyes-Ruiz, M., \& Stepinski, T. F. 1997, MNRAS, 285, 501

Roberts, P. H., \& Soward, A. M. 1975, Astron. Nachr., 296, 49

Rüdiger, G., \& Pipin, V. V. 2000, A\&A, 362, 756

Ruzmaikin, A. A., Shukurov, A. M., \& Sokoloff, D. D. 1988, Magnetic Fields of Galaxies (Kluwer, Dordrecht)

Sanders, D. B., Solomon, P. M., \& Scoville, N. Z. 1984, ApJ, 276,182

Sarson, G. R., Jones, C. A., Zhang, K., \& Schubert, G. 1997, Science, 276, 1106

Sarson, G. R., Jones, C. A., \& Zhang, K. 1999, Phys. Earth Planet. Int., 111, 47

Sciama, D. W. 1994, in Cosmical Magnetism, Contributed Papers in Honour of Prof. L. Mestel, ed. D. Lynden-Bell (Inst. Astron., Cambridge)

Sofue, Y. 1987, PASJ, 39, 547

Sofue, Y., \& Fujimoto, M. 1987, PASJ, 39, 843

Sokoloff, D., \& Shukurov, A. 1990, Nature, 347, 51

Vainshtein, S. I., \& Cattaneo, F. 1992, ApJ, 3393, 165

Zeldovich, Ya. B. 1956, JETP, 31, 154

Zeldovich, Ya. B., Ruzmaikin, A. A., \& Sokoloff, D. D. 1983, Magnetic Fields in Astrophysics (Gordon and Breach, New York) 